\title{
Reference
}

NBS

Publications

NAT'L INST. OF STAND \& TECH

NBSIR $84-2814$

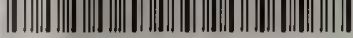

Al1106 टь1785

\ Survey of Sensitivity Analysis Methodology

U.S. DEPARTMENT OF COMMERCE

National Bureau of Standards

Center For Applied Mathematics

Operations Research Division

Washington, DC 20234

February 1984

Sponsored by:

U.S. DEPARTMENT OF COMMERCE

National Bureau of Standards

National Engineering Laboratory

Center for Applied Mathematics

Washington, DC 20234 


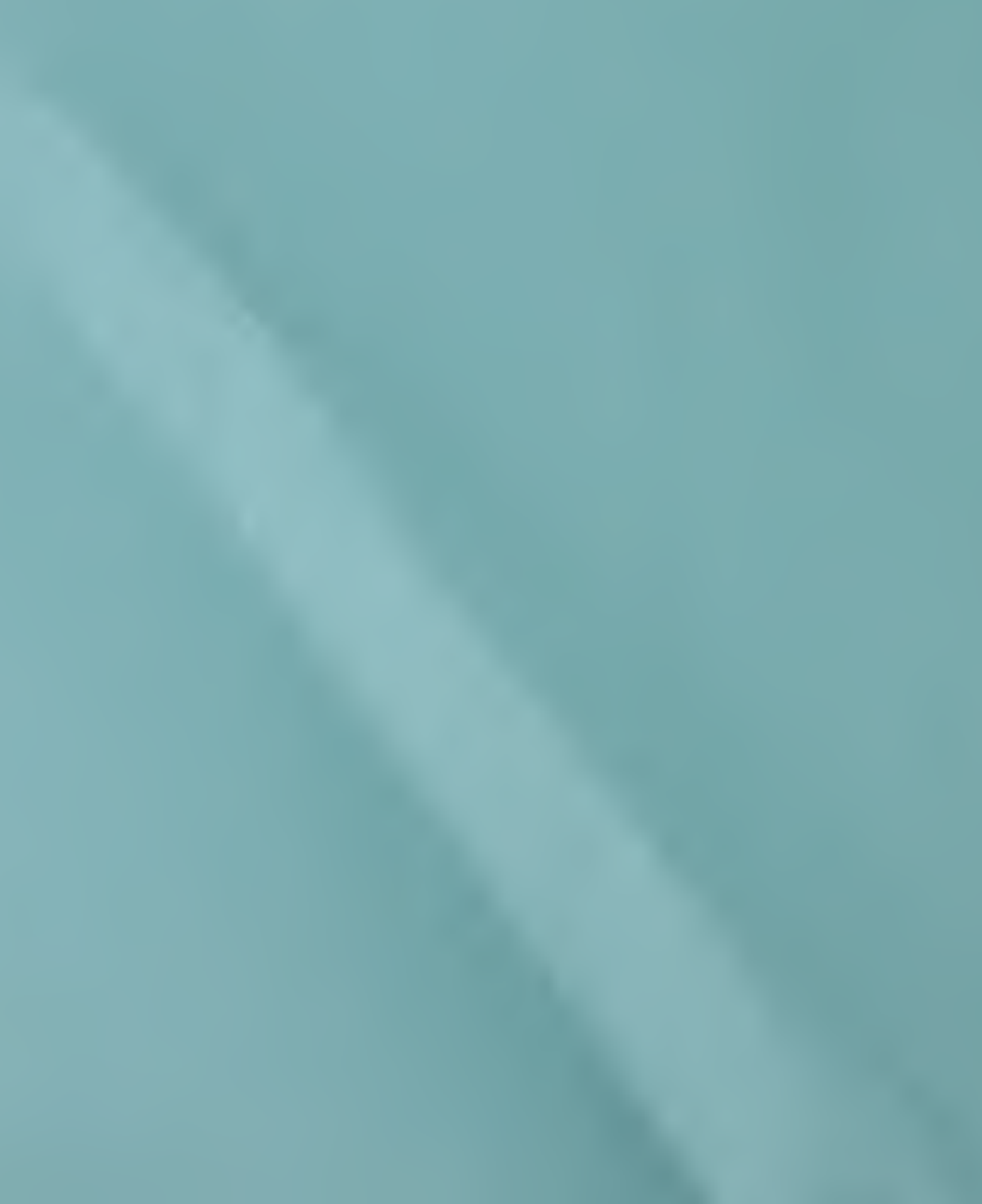




\section{A SURVEY OF SENSITIVITY ANALYSIS METHODOLOGY}

Robert G. Hendrickson

U.S. DEPARTMENT OF COMMERCE

National Bureau of Standards

Center for Applied Mathematics

Operations Research Division

Washington, DC 20234

February 1984

Sponsored by:

U.S. DEPARTMENT OF COMMERCE

National Bureau of Standards

National Engineering Laboratory

Center for Applied Mathematics

Washington, DC 20234

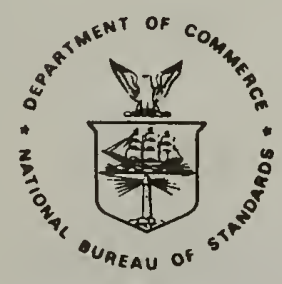

U.S. DEPARTMENT OF COMMERCE, Malcolm Baldrige, Secretary NATIONAL BUREAU OF STANDARDS, Ernest Ambler, Director 
1.0 INTRODUCTION

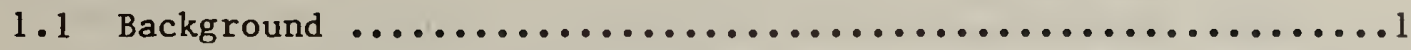

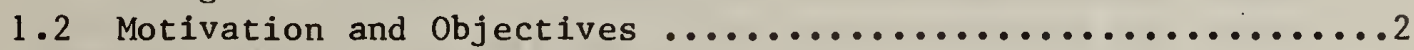

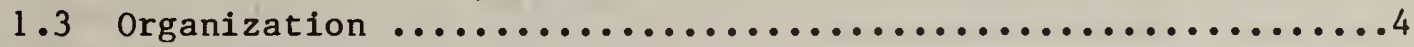

2.0 ERROR TYPES AND MODEL STRUCTURES

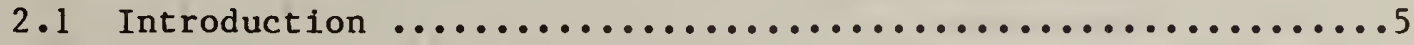

2.2 Error Types ...................................

2.2 .1 Accuracy of Numerical Values..................

2.2 .2 Process Errors ............................. 6

2.2 .3 System Errors $\ldots \ldots \ldots \ldots \ldots \ldots \ldots \ldots \ldots \ldots \ldots \ldots \ldots \ldots \ldots \ldots \ldots$

2.2 .4 Computational Errors $\ldots \ldots \ldots \ldots \ldots \ldots \ldots \ldots \ldots \ldots \ldots$

2.2.5 Errors of Sensitivity Analysis ................

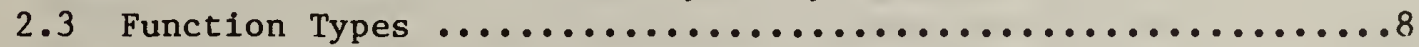

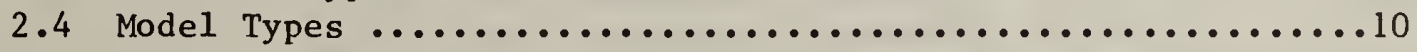

3.0 CLASSICAL METHODS OF SENSITIVITY ANALYSIS

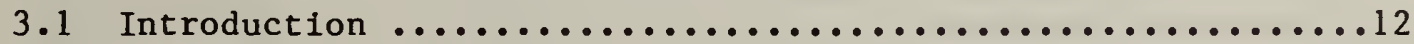

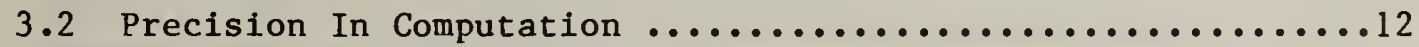

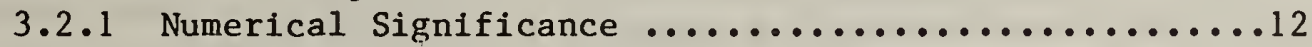

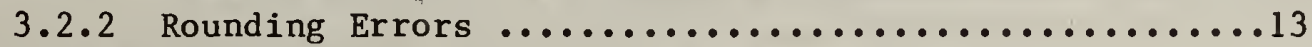

3.3 General Formulations of Error Analysis ............... 14

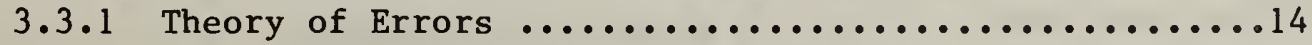

3.3.1.1 Quotient and Product Analysis ...........14

3.3.1.2 Integration, Interpolation,

Differentiation Errors ...............16

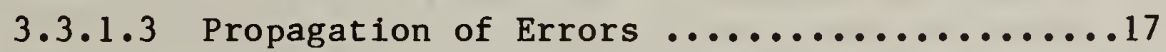

3.3.1.4 Differential Formulation of Error ........19

3.3.1.5 Variational Formulation of Error ........20

4.0 STATISTICAL METHODS OF SENSITIVITY ANALYSIS

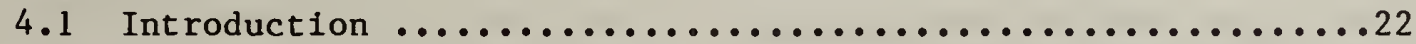

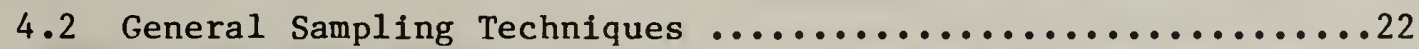

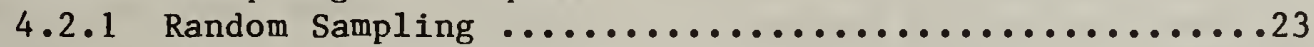

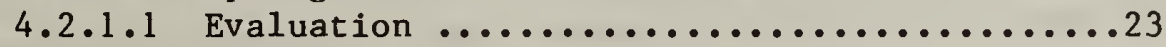

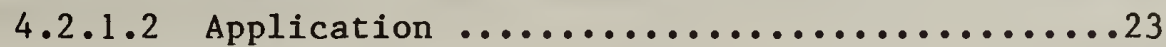

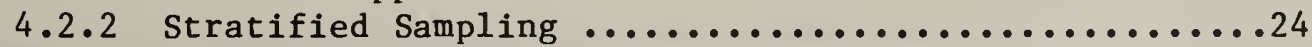

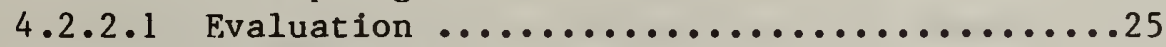

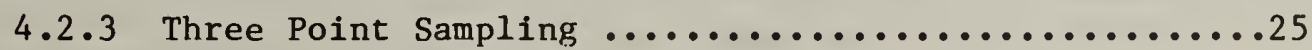

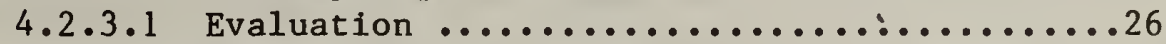

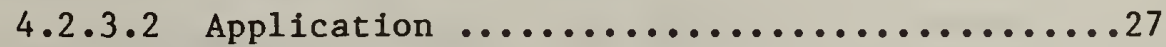

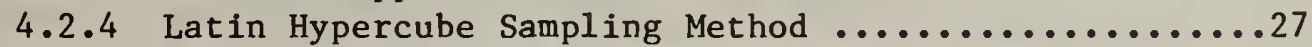

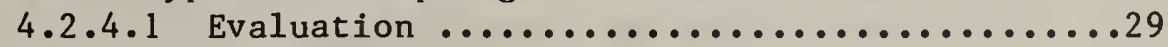

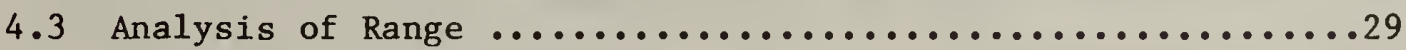

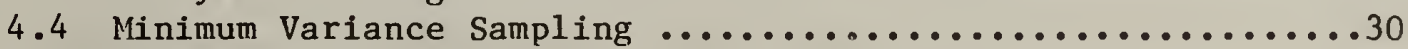

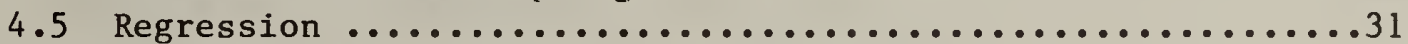

4.6 Partial Rank Correlation Coefficient (PRCC) $\ldots \ldots \ldots \ldots \ldots \ldots . . . .32$ 
5.0 ANALYTICAL METHODS OF SENSITIVITY ANALYSIS

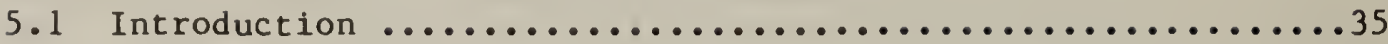

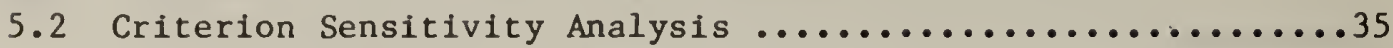

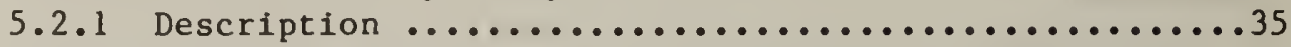

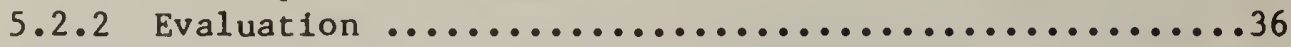

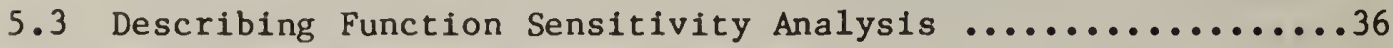

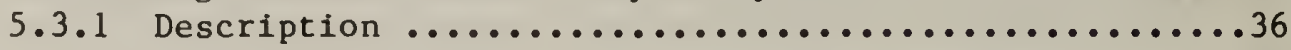

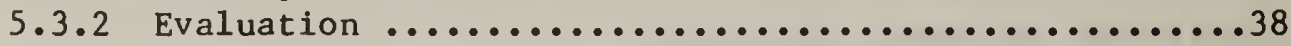

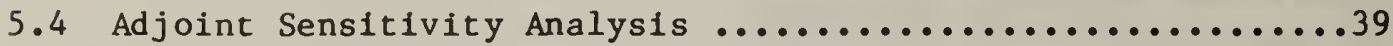

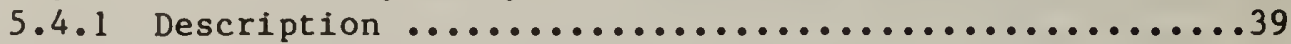

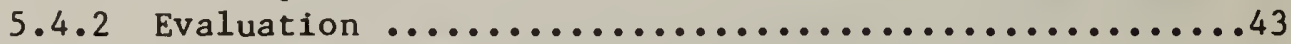

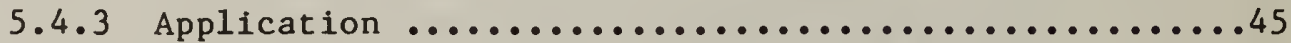

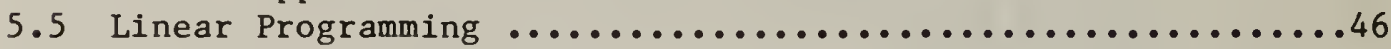

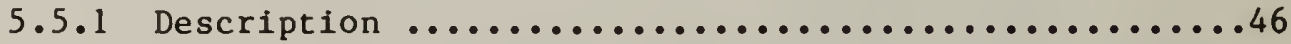

5.5.1.1 Postoptimality Evaluation $\ldots \ldots \ldots \ldots \ldots \ldots . \ldots 48$

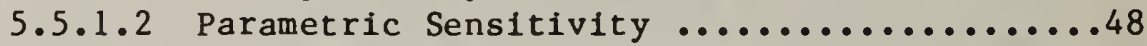

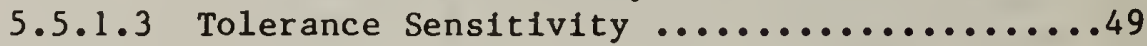

5.5.1.4 Lagrangian Parametric Sensitivity ........49

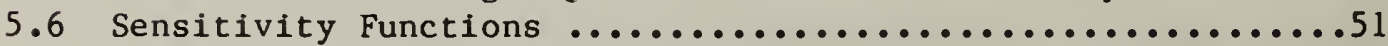

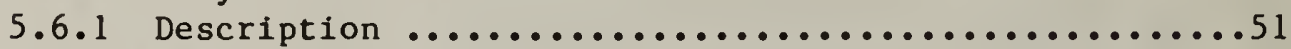

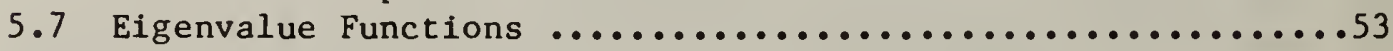

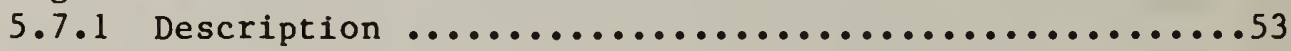

5.8 Large Error Sensitivity Analysis ....................... 54

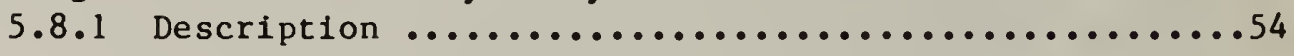

6.0 SPECIAL ANALYTICAL METHODS OF SENSITIVITY ANALYSIS

6.1 Introduction ................................. 56

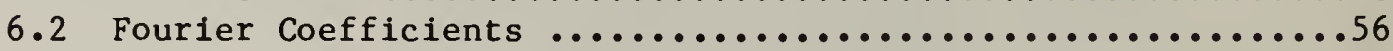

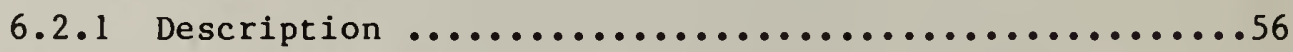

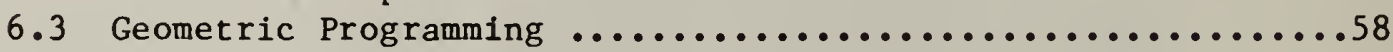

6.3 .1 Description ............................... 58

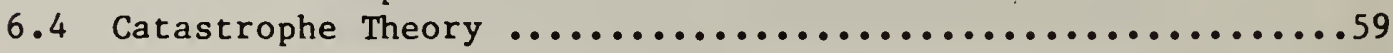

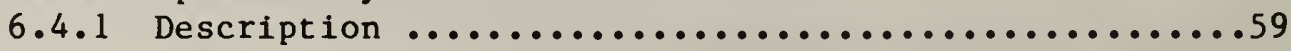

7.0 MODELING AND SENSITIVITY ANALYSIS

7.1 Introduction ..................................62

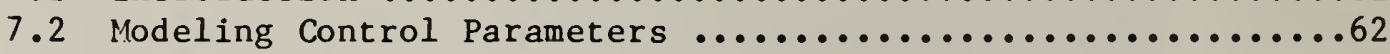

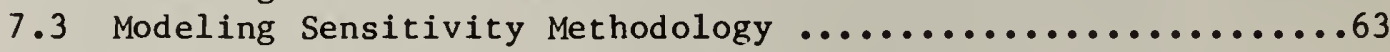

Append $1 x$ A ...........................................67

Part I Summary of Principal Error Formulas $\ldots \ldots \ldots \ldots \ldots \ldots \ldots \ldots \ldots 7$

Part II Statistics of the Index $w=x / y \ldots \ldots \ldots \ldots \ldots \ldots \ldots \ldots \ldots$

Appendix B: Parametric Programming: Sensitivity Analysis .........72

Appendix $\mathrm{C}$ : Catastrophe Flags and System Dynamics ..............75

References ..........................................79 


\subsection{INTRODUCTION}

\subsection{Background}

Sensitivity analysis is receiving a widening interest because of its role in model validation and the need to evaluate model results in terms of variations of input.

The complexity and sophistication of contemporary models and the attendant justification of model results in matters of policy and planning have created a demand for a wide range of sensitivity methodology.

Classical sensitivity analysis, which was formalized in the last century, is no longer adequate to meet the requirements of most modelling tasks. It was based either on an application of statistical methodology to observed data or on the use of differentials of variables of interest. These approaches are still used, particularly in experiments of an engineering nature or in the context of a controlled laboratory protocol, but they do not meet the needs, for various reasons which will be discussed, of contemporary model designs intended to be run on high-speed computers. The technical literature is reporting new analytical and statistical methods for validation and sensitivity analysis. These methods employ techniques which were developed specifically for the complex models of policy and allocation problems.

Sensitivity analysis is an important subject in model evaluation because it (1) aids in validating a model by contributing to an understanding of the correspondence between a model and its subject, (2) assists in the identification of variables which contribute only marginally to functional 
relationships among variables or elements in the model, (3) measures the quantitative impact of variations of input on model results, (4) tests the sensitivity of the level of representation of activities in the model to variations which effect actual perturbations or changes, and (5) contributes to measuring the degree to which input data may be altered without significantly exceeding a specified range-of-interest in the output.

A sensitivity analysis of a computational process or model may have to deal with: (1) errors which are inherent; that is, they are assumed to exist because of sampling or collection protocols (2) errors which vary stochastically; and (3) errors which are imposed upon the process by external circumstances or controls. The first kind of error, those which are embedded in the data, contribute to errors in the output primarily as displacements or scaling factors. The second kind of error, those which are random, project or convolute their randomness on the output, thereby giving the output the character of a statistical distribution. The third kind of error, that which is imposed, creates a displacement error in the output. The imposed error is evaluated by methods used to ascertain system biases. The advanced methodology of sensitivity analysis for evaluating these error types are drawn from specialized applications of mathematics, physics, and statistical sampling.

\subsection{Motivation and Objectives}

Sensitivity, in the sense of responses to variations, is of two broad kinds: qualitative and quantitative.

Qualitative sensitivity is used in this paper to denote the capability of a model to respond to dynamic changes in the subject being modeled. A closely related definition is the level of representation of the subject by its model. 
The sensitivity referred to here is the difference between the representation and the subject, and the significance of that difference in terms of second and third order effects.

An example of dynamic qualitative sensitivity would be a model of a queueing problem in which the logic of the model permits a dynamic reponse to queue sizes (demand) by opening or closing channels (service) according to the circumstances. Another example, though not commonly used in typical model design, is a heuristic model which alters its process based on a "learning" ability or the use of artificial intelligence to select or alter its logic according to accumulated information. The use of "dynamic" should not be confused with an association of time as a model variable, although it is not excluded from the discussion above - dynamic, as used here, refers to the ability of the model to automatically modify its logic or computational process according to critical perturbations in specified state variables which may or may not be functions of time. Most models deal with a specified scenario with almost no capability to simulate potential system dynamics. It is, therefore, difficult to validate these models or to understand thoroughly their range of application.

Quantitative sensitivity is defined as the numerical measure of changes in output to variations of input. This is the traditional view of sensitivity analysis.

The motivation for preparing a survey of sensitivity methodology addresses both the need to recognize and analyze qualitative and quantitative sensitivity problems. The qualitative aspect addresses model design; the quantitative aspect addresses responses to parametric variation. 
The motivation and objectives of this survey are interlinked in the need and desire to provide sensitivity methodology and reference in a single source; to advertise to the NBS staff, and others, the importance of the subject; to improve the understanding of sensitivity analysis in the design of models; and to enhance the ability to perform a more efficient model validation through the use of qualitative and quantitative sensitivity methodology.

This survey was written to provide a general overview of sensitivity analysis to the model developer and is not intended to provide a precise theoretical and comprehensive treatment of the methods included in the survey. 1.3 Organization

The paper is organized into six sections which develop and evaluate the classical and modern methods of sensitivity analysis. Section 2.0, Error Types and Model Structures, defines the error types incurred in modeling, and in statistical and physical experiments; Section 3.0, Classical Methods of Sensitivity Analysis, presents the familiar theory of errors and numerical significance; Section 4.0, Statistical Methods of Sensitivity Analysis, describes the basic statistical approaches to measuring model performance and other aspects of statistical interest; Section 5.0, Analytical Methods of Sensitivity Analysis, presents an assortment of methods which constitute the major advances in different lines of sensitivity methodology; Section 6.0 , Special Analytical Methods of Sensitivity Analysis presents some very interesting techniques and concepts for certain kinds of sensitivity problems with broad application; and Section 7.0, Modeling and Sensitivity Analysis, in which methods of model assessment and design concepts are presented and evaluated. 
Although it may not be possible to discuss every method presented with equal knowledge or to know the consequences of its application, an attempt to evaluate some of the methods has been made. The evaluation, when given, is based on information from cited references which includes comments on efficiency, ease of use, cost, preparation, sample size, and output description.

Equations cited within quoted material retain their source designations; equations in the text are numbered according to the major section in which they appear. 


\subsection{ERROR TYPES AND MODEL STRUCTURES}

\subsection{Introduction}

This section introduces the basic types of errors incurred in data and model analysis. It presents the context of these error types by discussing generic functional forms on which sensitivity analysis is carried out, and rounds out the discussion of these subjects with a description of model types and their relation to sensitivity analysis. Taken together these three subjects define fundamental relationships among the model, the representation of the subject, and the data. It is from these relationships that data and model uncertainties have their origins.

\subsection{Error Types}

There are five major types of errors ranging from the accuracy of input data to the implicit errors introduced by the selection of computational methods, algorithms, and the formulas themselves which are used to evaluate model sensitivity.

\section{2 .1 Accuracy of Numerical Values}

Although there may be many ways in which uncertainties can be incorporated into input data, the error which is emphasized here is that of the number of significant digits in data of a cohort or homogeneous file. Significance of numbers is a field of numerical analysis which has been studied for a long time and most of the rules addressing numerical precision are well known. Several of these rules will be summarized here to refresh our 
recognition of the subject and to refer to these rules when our examination reaches the study of the propagation of errors in a computatonal process. Following Reference [1], Kelly gives three general rules dealing with numerical precision:

(1) If the first significant figure of a number is $k$, and the number is correct to $n$ significant figures, then the relative error in this number is less than

$$
\frac{1}{\mathrm{k} 10^{\mathrm{n}-\mathrm{I}}}
$$

where the relative error is defined as the error divided by the number.

(2) If the relative error in a number is less than

$$
\frac{1}{(k+1) 10^{n-1}}
$$

the number is correct to $n$ significant figures or is in error by less than a unit in the nth significant figure.

(3) A number is correct to $n$ significant figures if the relative error of the number is not greater than

$$
\frac{1}{2 \cdot 10^{n}}
$$

The propagation of errors in significant digits depends entirely on the distribution of the errors throughout the data set and the computational process to which the data are subjected in the model.

\subsubsection{Process Errors}

Process errors are those errors introduced by rounding and by truncation. These errors arise primarily from register lengths in computer hardware, and are entirely dependent on the mechanical features of the equipment used for 
the model computation. They may or may not be important to sensitivity analysis since most computers provide floating point calculations. It is not unreasonable to make the point that, for most simulations, the input data are far less accurate than the capacity for accuracy provided by most modern computers. However, round-off error is an extremely important subject and has been treated widely in the literature as it relates to and impacts on the accuracy of repeated calculations, convergence criteria, and decisions based on numerical differences.

\subsubsection{System Errors}

There are several categories of errors which can be thought of as associated with system processes: those that come from system bias, those that arise from system noise, those that originate from measurement, those that occur in data generation protocols, and those that arise from data sampling. These may be regrouped under three more convenient categories: systematic errors; to cover system bias and noise; measurement errors, to cover the errors of measurement, sampling, and generation; and structural errors, to describe errors imposed by the computational process of the model. It is necessary to examine the possibility that the structure of the model or experimental design may directly affect the results of an experiment. This point involves both qualitative sensitivity and quantitative sensitivity; it is an important aspect of sensitivity analysis because model design may Impose an error of perception or bias on the structure of the model. The general theory of system errors, as they occur in the context of physical experiments, is nicely covered in References [3], [4], and [5]. 


\subsubsection{Computational Errors}

In this class of errors there are three levels at which qualitative or quantitative errors are likely to be incurred. A qualitative error is an error imposed by selecting a particular method of computation or representation; and a quantitative error is an error associated with numerical calculations.

The first level the source of error is the method of computation, representation, or degree of aggregation of the subject. By choosing a particular process for a computation, approximations to the subject are automatically imposed which invest the process with attendant quantitative errors. The choice of algorithm or model logic is a qualitative decision, but when the choice is made, quantitative errors are imposed on the results obtained from the method of computation.

The second level of error is that which originates from the equations used by the selected method of computation. This level of error is characterized by the calculation of quantitative errors in the form of relative functional errors, sensitivity coefficients (discussed in detail in 3.3.1.4), and parametric variations.

The third level of error is the error of calculation, which results from a sequence of arithmetic operations upon numbers in the equations of the method of computation. This level addresses issues of significance of the input data, significance of results of arithmetic operations, and the net precision of the final result of the computational process. Each level contributes to the propagation of errors in either the quantitative or qualitative sense, or both. 


\subsubsection{Errors of Sensitivity Analysis}

The methods of sensitivity analysis, whether they be statistical or variational formulations, or any of the many forms described in this report, may themselves create errors because some are approximations or, by the argument of Section 2.2.4 above, they may induce errors or biases because they involve sequences of calculations.

The methods of quantitative sensitivity analysis are primarily either statistical in nature, use local approximations (Taylor series), or employ a closed functional solution to obtain the desired sensitivity coefficients. There also may be assumptions which bear on numerical characteristics, such as distribution or error magnitude, which contribute to the total quantitative sensitivity of a model's computational process.

\subsection{Function Types}

Error analysis is ultimately contingent on the nucleus of arithmetic operations embedded in a method, a model, or an experiment. These operations collect and accumulate qualitative and quantitative errors which are the pursued objects of sensitivity analysis. A model is often, but simplistically, described as a function, as in

$$
y=f(a, x)
$$

where the " $a$ " is a set of input control data and " $x$ " is a set of input state data. The model is represented by the operator " $f$ " which acts on " $a$ " and " $x$ " to produce output " $y$ ". Equation $(2.4)$ is the basic representation which is used in sensitivity analysis for the development of absolute and relative errors in the variable $y$.

An important extension of this concept is the system which involves embedded functional relationships, as in

$$
\begin{aligned}
& y=f(a, x) \\
& u=g(b, y, v)
\end{aligned}
$$


in which "b" is a set of control input data, $y$ is the output from the previous equation, "v" is a set of control state variables which may include some subset of " $\mathrm{x} "$, and " $\mathrm{g} "$ is an operator. This process of chaining is common to most simulations and models of any degree of complexity.

The next step up in functional formulation is the system of simultaneous equations, given as

$$
\mathrm{y}=\mathrm{Ax}
$$

for a linear system, where $\mathrm{A}$ is a square matrix and $\mathrm{x}, \mathrm{y}$ are vectors; or as

$$
y^{\prime}=f(x, y)
$$

the differential form, with $x, y$ as vectors and $y^{\prime}$ as the derivative of $y$ with respect to $x$. Equation (2.7) could also be expressed as

$$
y=f(x, y, t)
$$

where $y$ is the derivative of $y$ with respect to time, " $t$ ". Equation (2.8) is the general form for the sensitivity function, discussed below in Section 5.6. Equation (2.6) appears in many problem formulations and most particularly as the basic form, with an objective function, of a linear program. If in equation (2.6) $y=\lambda x$, then the familiar eigenvalue formulation emerges.

2.4 Model Types

It is customary to refer to model types as descriptive, prescriptive, or predictive, and within these, the models as being static or dynamic. These categories designate purpose and structure, but they are not particularly useful distinctions for the purposes of sensitivity analysis. 
As this Section has attempted to indicate, there is a progression of error analysis which spans the numerical significance of data at the lowest level to the extensive variety and complexity of models and simulations at the highest level. At each stage of this progression the complexity of the error analysis increases in degree over the previous stage, which requires, in turn, an increase in the sophistication of the sensitivity methodology as the progression moves from simple to complex model processes.

Many of the methods of sensitivity analysis have been developed to evaluate sophisticated models of complex subjects, and therefore are, in many cases, designed for a particular treatment or mathematical formulation. There are, then, at least seven general types of model processes that can be identified; and each has its own particular method of sensitivity analysis: (1) independent algebraic or differential equations, (2) chained dependent algebraic equations, (3) system of algebraic equations (4) system of differential equations, (5) system of stochastic differential equations, (6) statistical distribution functions or variables, and (7) the stochastic or deterministic iterated, closed program (algorithm). There are certainly many other variations of these types that could be listed, but to dwell on categories would obscure the principal point, namely, that sensitivity methodology consists of general techniques for analyzing subject formulations rather than analyzing specific model types. Even the model itself, because of its organization of components, degree of complexity, and level of aggregation may be considered as a process which is appropriate for sensitivity analysis as a part of the larger process of validating subject-model correspondence. 
Model types, therefore, which relate to sensitivity analysis should be categorized in terms of the representation of the subject. This concept is essentially the reverse of the conventional approach, which is to identify the model by the purpose of the model and the use of its output. 


\subsection{Introduction}

The principal subjects associated with classical sensitivity analysis are computational precision (primarily rounding errors and numerical error estimation), the general theory of errors (addressing variations of functions and products or quotients of random variables), and differential formulations and approximations (which provide closed, analytical expressions of error and sensitivity). These subjects are examined in the following sections. The material is not intended to be exhaustive but rather in the spirit of a survey of issues of primary interest to model developers rather than to numerical analysts.

\subsection{Precision In Computation}

Computational precision is represented primarily by the net numerical significance of arithmetic calculations and by the analysis of roundoff errors in computational operations.

\subsubsection{Numerical Significance}

Section 2.2.1, Accuracy of Numerical Values, introduced several rules about relative errors in numbers and measures of these errors. There are two ways of defining a significant figure: (1) as any of the ten digits, except zeros which are used only to locate the decimal point, and (2) any digit which signifies the amount of quantity in the place in a number in which it stands. We now state from Kelly, Reference [1], the relation of significance to simple arithmetic operations.

(1) If two numbers are added or subtracted the maximum error is the sum of the maximum errors of the two numbers,

(2) If two numbers are multiplied or divided, the result has a maximum relative error equal to the sum of the relative errors of the two numbers. 
In addition, experience has shown that errors of a sum or multiplication compensate to a degree, especially in a long string of calculations. The loss of significance by subtraction is the principal source of error.

Additional rules advise on accuracy where a variation is introduced:

(3) retain enough significant figures in a number to include the place in which the least significant figure of the variation occurs; and

(4) in adding or subtracting several numbers deal with absolute error, not the relative error,

(5) when several values are to be multiplied or divided use the largest relative error in computing the number of significant digits of the result. Stoer \& Bulirsch, Reference [2], have written a comprehensive and modern treatment of computer arithmetic, error propagation and interval analysis, which complements the older texts on numerical analysis.

\subsubsection{Rounding Errors}

This subject is usually included under discussions on computational precision where the roundoff is made to a number in a particular digit of the number, rounded up or down in that digit, and the rest of the number is truncated. There is, however, a more sophisticated analysis of the errors associated with rounding, which is given in Reference [2], Chapter 1, and is there referred to as statistical roundoff estimates. This concept defines the relative roundoff error which results from an elementary operation as a random variable on a given interval of values. The roundoff errors are assumed to be independent with a given distribution of values. Seen in this way, the evaluation of roundoff errors proceeds along classical statistical 
determinations of the means and variances of results of computations. The assumption of independence is critical: if the roundoff errors are not independent the error analysis requires a more complicated formulation.

\subsection{General Formulations of Error Analysis}

This section presents the subjects which comprise the bulk of classical sensitivity analysis. The familiar results of numerical analysis, statistical theory and error estimates are developed and evaluated. These methods, and the statistical methods described in Section 3.3 .2 below, are the most widely used of all the techniques available to model assessors. A summary of the principal formulas of the propagation of errors and classical sensitivity analysis is provided in Appendix A.

\subsubsection{Theory of Errors}

Within this broad subject there are three subordinate subjects which are discussed separately in order that their contributions to the general theory of errors are clearly delineated. These three subjects are product and quotient analysis; errors of integration, interpolation and differentiation; and propagation of errors.

\subsubsection{Quotient and Product Analysis}

There are two basic relationships in the theory of errors which are fundamental to sensitivity analysis. These relationships are the statistical expression of the means and standard deviations of the product and quotient of two random variables. 
Given that $\mathrm{x}$ and $\mathrm{y}$ are random and normally distributed with means $\mu_{\mathrm{x}}$ and $\mu_{y}$, and standard deviations $\sigma_{x}$ and $\sigma_{y}$, let

$$
\mathrm{w}=\mathrm{x} / \mathrm{y},
$$

then the mean of $w, \bar{w}$ is:

$$
\mathrm{w}=\mu_{\mathrm{x}} / \mu_{\mathrm{y}}, \quad \mu_{\mathrm{y}} \neq 0
$$

The standard deviation of $w$ was developed in a basic paper by Fieller [6], and is as follows: If $w=x / y$, and $x$ and $y$ are independent and normally distributed with means $\mu_{\mathrm{x}}$ and $\mu_{\mathrm{y}}$ and standard deviations $\sigma_{\mathrm{x}}$ and $\sigma_{\mathrm{y}}$, then $w$ will be approximately normally distributed with mean given in (3.2) above and a standard deviation of :

$$
\sigma_{w}=\frac{\mu_{x}}{\mu_{y}}\left[\frac{\sigma_{x}^{2}}{\mu_{x}^{2}}+\frac{\sigma_{y}^{2}}{\mu_{y}{ }^{2}}-2 r \frac{\sigma_{x} \sigma_{y}}{\mu_{x} \mu_{y}}\right]^{1 / 2}
$$

where $r$ is the correlation coefficient and $\mu_{y} / \sigma_{y}>5$. See Appendix A, Part II, for more details on this theory.

Craig [7] developed a similar formula for the product of random variables

$$
\mathrm{w}=\mathrm{xy}
$$

If $x$ and $y$ are independent and normally distributed with means $\mu_{x}$ and $\mu_{y}$, and standard deviations $\sigma_{x}$ and $\sigma_{y}$, then the mean of $w$ is

$$
\bar{w}=\mu_{x} \mu_{y},
$$

and the standard deviation is:

$$
\sigma_{w}=\left(\mu_{x}^{2} \sigma_{y}^{2}+\mu_{y}^{2} \sigma_{x}^{2}+\sigma_{x}^{2} \sigma_{y}^{2}\right)^{1 / 2}
$$

In the case that $x$ and $y$ are not independent, but normally distributed, $\sigma_{w}$ is (from [7], page 9):

$$
\sigma_{w}=\left[\mu_{x}^{2} \sigma_{y}^{2}+\mu_{y}^{2} \sigma_{x}^{2}+2 r \mu_{x} \mu_{y} \sigma_{x}{ }^{2}+\sigma_{x}^{2} \sigma_{y}^{2}\left(1+r^{2}\right)\right]^{1 / 2}
$$


where $\mathbf{r}$ is the correlation coefficient. Reference [8] gives a procedure for obtaining either one-sided or two-sided tolerancel limits of the product and quotient of independent normal variates. It is assumed in this procedure that the distributions have positive means and small coefficients of variation $\left(\sigma_{x} / \mu_{x}\right)$. Huntington [9] derives frequency distributions for the product and quotient of two independent random variables, and Curtiss [10] provides a general and thorough development of the frequency and distribution functions of the quotient of two random variables. (Curtiss concludes his paper with a brief treatment of the distribution of the product of two random variables.) His theorems are generalized and expressed as integral functions depending on the positive and negative domains of the product.

\subsubsection{Integration, Interpolation, Differentiation Errors}

These mathematical methods are well known and thoroughly treated in texts on numerical analysis. The advent of high-speed, sophisticated computers has made it possible to build models of extreme complexity. This capacity has required a new development of numerical procedures, and as a consequence the classical numerical techniques have been revitalized or replaced by advanced, innovative methods.

No attempt will be made here to restate the classical methodology nor will any attempt be made to reproduce any of the many advanced numerical techniques except to recognize their existence and their immense importance to many diverse areas of application. Software packages abound which contain

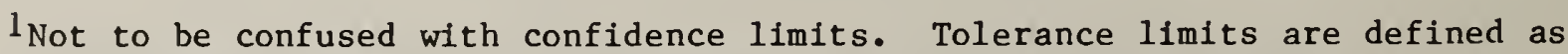
the limits which include at least "p" percent of the observations at some prescribed level of confidence.
} 
specific and generic numerical tools for virtually every computational need, and the underlying theory of these developments have given numerical analysis an enhanced and extensive revitalization.

\subsubsection{Propagation of Errors}

The theory of propagation of errors should be more properly called the theory of small errors. It was developed about a century ago and most of the early texts which appeared around the turn of the century dealt with analysis of observations generated from physical experiments and calibration of instruments. More recently Ku [12], Dietrich [5], and Deming [13] have written more comprehensive treatises on experimental error and provided a sound theoretical basis for their formulations. Of these modern treatments Ku has written the most complete and thorough exposition of the propagation of errors. In his paper, Ku states two forms the analysis of random functions can take: (1) determining the statistical parameters of a given function of a random variable, or (2) determining the statistical parameters in which the function is assumed to tend, asymptotically with a large number of observations, to the normal distribution. Ku's paper deals only with the second formulation of the problem. The problem, where the function or its expected variation does not, for whatever reason, conform to asymptotic normality is of equal importance, however, and should be addressed. Section 5.9 will address the various methods which can be used where the assumption of asymptotic normality is not essential.

The basis for the traditional approach to the study of errors is embodied in a theorem, which is paraphrased from $\mathrm{Ku}$, as follows:

If, in some neighborhood a function of random variables is continuous and possesses continuous first and second derivatives (i.e. $f(x) \varepsilon C^{2}$ ), then the statistical parameters of the function can be approximated by 


$$
\overline{f(x)}=\text { expectation of } f(x) \cong f(\bar{x}) \text {, }
$$

and the variance is given by

$$
\operatorname{var} f(x) \cong \frac{1}{n} \sum_{i=1}^{n}\left(\frac{\partial f}{\partial x_{i}}\right)^{2} \sigma_{x_{i}}^{2}+\frac{1}{n} \sum_{p=1}^{n} \sum_{q=1}^{n}\left(\frac{\partial f}{\partial x_{p}}\right)\left(\frac{\partial f}{\partial x_{q}}\right){ }^{\sigma} p q
$$

(where $\mathrm{x}$ is a vector of $\mathrm{n}$ elements) based on asymptotic normality. A Taylor series expansion, from which second and higher order terms are dropped, of the function over the continuous neighborhood serves a basis for the derivation of the statistical approximations to the mean and variance, as given by equation (3.8) and (3.9). There are, then, three conditions attendant on the acceptance of this development (1) that the variations of the independent variables are small enough to justify ignoring second and higher order terms from the Taylor series expansion, (2) that the approximations of statistical parameters tend to the normal as the number of observations becomes large, and (3) that the function is necessarily well behaved in the domain of interest. The case of large error or small sample size is not amenable to the above conditions, and therefore requires its own methodology. The use of any of the familiar approximations, which measures error or computes a statistical parameter, introduces an error (induced qualitative error ${ }^{l}$ ) the size of which depends on the formula employed, the number of observations, and the magnitude of the uncertainties in the independent variables of the function. The main references, [5], [12], [13], and the subsidiary references [14-20] provide a complete statement of the theory of small errors. Of particular interest is Ku's extension of the theory to accuracies of the various stages of approximations.

${ }^{1}$ See Section 2.2 .4 


\subsubsection{Differential Formulation of Error}

An alternative formulation to the theory given above is to require the function to be only of function class $\mathrm{Cl}^{l}$, i.e., needing only a continuous first derivative, and expand the function as a total differential, and use this result as a measure of absolute sensitivity. Let $f(x)$, where $x$ is a vector, be $\mathrm{c}^{l}$, then

$$
\mathrm{df}=\sum_{i=1}^{n} \frac{\partial f}{\partial x_{i}} d x_{i}
$$

where the $x_{i}$ are independent or are functions of another variable. If the function is defined as

$$
w=f(u, v)
$$

where $u$ is a vector of control variables and $v$ a vector of state variables, and $u_{i}$ is one of the $u\left(u_{i} \varepsilon U\right)$, the sensitivityl to $u_{i}$ may be developed as follows :

$$
d w=\sum_{j=1}^{n} \frac{\partial f}{\partial u_{j}} d u_{j}+\sum_{h=1}^{m} \frac{\partial f}{\partial v_{h}} d v_{k}
$$

and forming $d w / d u_{i}$, we obtain

$$
\frac{d w}{d u_{i}}=\frac{\partial f}{\partial u_{i}}+\sum_{\substack{j=1 \\ j \neq i}}^{n} \frac{\partial f}{\partial u_{j}} \frac{d u_{j}}{d u_{i}}+\sum_{k=1}^{m} \frac{\partial f}{\partial v_{k}} \frac{d v_{k}}{d u_{i}}
$$

as the sensitivity of the function $w$ to variation in $u_{i}$. The first order partial derivatives in equation (3.14) are called sensitivity coefficients in the literature. The rates $d u_{j} / d u_{i}$ express the functional dependencies among the control elements on $u_{i}$. The rates $d v_{k} / d u_{i}$ express the functional relationships, if any, of the state variables with the $u_{i}$ control variable.

${ }^{1}$ In some models it may be important to identify control and state variables in order to refine the evaluation of a sensitivity analysis. In this example $u$ and $v$ represent this distinction. 
If, in equation (3.14) we are interested in time dependent changes in $w$, the equation becomes:

$$
\dot{w}=\frac{d w}{d t}=\sum_{i=1}^{n} \frac{\partial f}{\partial u_{i}} \frac{d u_{i}}{d t}+\sum_{k=1}^{m} \frac{\partial f}{\partial v_{k}} \frac{d v_{k}}{d t} \text {; where } \frac{d u_{i}}{d t}=\dot{u}_{i} \text { and } \frac{d v_{k}}{d t}=\dot{v}_{k}
$$

\subsubsection{Variational Formulation of Errors}

In analogy with the definition of the differential, we define $\delta$ as the variation in a function or variable. From Hildebrand [21], the differential of a function is an approximation to the change in that function along a particular curve, while the variation is a first order approximation to the change from curve to curve. The operators $\frac{d}{d x}$ and $\delta$ are commutative for independent variables, and are therefore interchangeable, so that

$$
\frac{d}{d x}(\delta y)=\delta\left(\frac{d y}{d x}\right)
$$

We can write the variational form of equation (3.10) as

$$
\delta f=\sum_{i=1}^{n}\left(\frac{\partial f}{\partial x_{i}}\right) \delta x_{i}
$$

The variation is usually defined as

$$
\delta \mathrm{x}=\varepsilon \phi(\mathrm{x})
$$

which changes a function, say $y(x)$, to a new function, $z(x)$ by:

$$
z(x)=y(x)+\delta x=y(x)+\varepsilon \phi(x),
$$

where $\varepsilon$ is some appropriate value.

Hence the values which $\delta x$ may take are not restricted. The statistical

approximation to the variance, as developed above in Section 3.3 .1 .3 , equation (3.10), can be derived from (3.21) by squaring $\delta \mathrm{f}$, taking the expected values 
of both sides of the equation and reducing the terms to their variance. Weld [14] has derived the more usual expression for the standard deviation:

$$
S=\left[\sum_{i=1}^{n}\left(\frac{\delta f}{\delta X_{i}}\right)^{2} \sigma_{i}^{2}\right]^{1 / 2}
$$

by this method. For this equation to be true, however, would require independence of the variables, as was required in the derivation based on Taylor series expansion. The $\delta \mathrm{x}$ would be restricted to represent the error or variation in the observations, and would, under the protocol of a large sample, comply with the requirements of convergence to normality. This condition is essentiat :o all of the formulations of classical error analysis. 


\subsection{STATISTICAL METHDDS OF SENSITIVITY ANALYSIS}

\subsection{Introduction}

This Section presents certain specialized applications and developments of statistical methodology which are used in the sensitivity analysis and evaluation of large models.

The methods discussed are sampling techniques, which are motivated by model evaluation problems; analysis of rangel data, which is applicable to parameter estimation and to the evaluation of large errors; the use of partial rank correlation coefficients as measures of sensitivity; and three standard statistical methods, rangel analysis, minimum variance, and regression, which are used for estimating and sizing sensitivity.

Of these subjects the sampling techniques and application of partial rank correlation coefficients as measures of sensitivity are of particular value to those interested in assessing a large, complex computerized model. The best of the sampling schemes, latin hypercube sampling, is a procedure which structures input trials in an optimum and comprehensive model-testing design. 4.2 General Sampling Techniques

Three principal methods of sampling are presented. Two of the three methods are familiar and are the subjects of a wide and comprehensive literature. The third method, however, is comparatively new, that of latin hypercube sampling (LHS), and is discussed and compared with standard sampling procedures.

l"range" is used here to refer to the extreme values of an interval around a value. 


\subsubsection{Random Sampling}

In random sampling, values for input variables are determined by a scheme which assigns a value equally likely over all the values the input variable is permitted to have. Further, the sample values so selected have the same probability of selection as any other set of assignments of values to the variables. The collection of values over all the variables is called the sample space. The sample then is the set of assigned values to each input variable under the condition that each assignment was as equally likely as any other assignment and that each assignment is independent from all other assignments.

4.2.1.1 Evaluation There exists an immense literature on the analysis of random sampling and its use in estimating the statistical parameters of the population from which the sample was taken. Our intent in introducing random sampling is to compare its efficiency against other methods of sampling, particularly as it relates to generating data for senstivity analysis and model evaluation. It will be shown below that a variance based on a random sample will be greater than or equal to the variance based on a stratified sampling scheme. See references [22] and [23] for technical exposition. 4.2.1.2 Application The use of random sampling is wide spread, and in the specified requirements of some models it has the desired properties needed to attain certain conditions, but as a technique for generating data for model evaluation it is the most costly and inefficient of the three methods outlined in this Section. 


\section{4.).) Stratified Sampling}

In stratified sampling the sample space of input variahles is partitioned into " subspaces. Following the development by McKay, Reckman, and Conover 123], the sample space is partitioned into disjoint sets $s_{i}$ of size $p_{\mathfrak{f}}$, and if $x_{i j}$ are a raminm sample $(i)$ on $s_{i}$, and with $y=h(x)$, an unknown but ohservahle mapping. then the strata means and variances of $\mathrm{g}(\mathrm{y})$ are:

$$
\begin{gathered}
\mu_{i}=F\left(g\left(y_{1 j}\right)\right)=\int_{S_{i}} g(y) \frac{1}{p_{i}} f(x) d x \\
\sigma_{i}^{2}=V\left(g\left(y_{i j}\right)\right)=\int_{S_{i}}\left(g(y)-\mu_{i}\right)^{2} \frac{1}{p_{i}} f(x) d x
\end{gathered}
$$

and the estimator over the sample space

$$
T=\frac{1}{N} \sum_{i=1}^{N} g\left(u_{i}\right),
$$

where $y\left(u_{i}\right)$ is an arbitrary, known function. Then

$$
T_{s}=\sum_{i=1}^{I}\left(\frac{P_{i}}{n_{i}} \sum_{j=1}^{n_{i}} g\left(y_{1 j}\right)\right.
$$

and the variance of $T_{s}$ is given by:

$$
V\left(T_{s}\right)=\sum_{i=1}^{I}\left(\frac{P_{i}^{2}}{n_{i}}\right) \sigma_{i}^{2},
$$

where the "s" on $\mathrm{T}$ as a subscript indicates "stratified sampling" and I is the number of disjoint subsets $s_{i}$. Invoking the development of Tocher [24], equation ( 4.5$)$ becomes:

$$
V\left(T_{S}\right)=V\left(T_{R}\right)-\frac{1}{N} \sum_{i=1}^{I} P_{i}\left(\mu_{i}-\tau\right)^{2}
$$

where " $R$ " on $T_{R}$ denotes the estimator based on rapidom sampling and $\tau$ is the estimate of the mean under stratified sampling. It follows from equation (4.6) that 


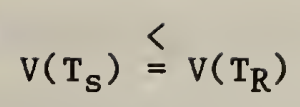

which is the desired demonstration that stratified sampling is a more efficient method than is random sampling. A necessary condition attendant to this conclusion is that the sample sizes, $n_{i}$, are chosen so that

$$
\mathrm{n}_{1}=\mathrm{P}_{1} \mathrm{~N}
$$

which provides for proportional allocation. The variance reduction shown in equation $(4.6)$ is a function of the differences between the strata means $\left(\mu_{i}\right)$ and the overall mean $(\tau)$.

4.2.2.1 Evaluation Although the advantage of a reduced variance is gained through the use of stratified sampling it is not certain that this method of sampling can be applied to large simulations. The sample size per cell, equation ( 4.8$)$, and the partitioning of the data set may be impossible to impose or may not be a natural realization of the system or subject which has been modeled. The amount of the reduction is also a factor to consider. In both the random and stratified sampling methods $N$ runs are made. If the cost of partitioning is substantial it must be measured against the value attained in the reduction of the variance. It follows, then, that a cost-benefit analysis imposes on the decision to use stratified sampling.

\subsubsection{Three Point Sampling}

This plan uses three values to represent the range of values for inputs. These values are assigned independently to each run based on a probability for each value, the relationship. being

$$
\mathrm{P}_{1}=\mathrm{P}_{2}=\frac{1}{2}\left(1-\mathrm{P}_{\mathrm{O}}\right)
$$


where $\mathrm{P}_{\mathrm{O}}$ is the probability of the median value which is assigned by the analyst. Under proportionate allocations $3\left(1-\mathrm{p}_{0}\right) \frac{\mathrm{n}}{2}$ values are assigned at random to $n$ runs of the model. For example if $x_{-}, x_{+}$, and $x_{0}$ are the lower, higher and median values of variable $x$ with probabilities $P_{1}, P_{2}$, and $P_{0}$, then a random draw based on an interval in proportion to the values of $P_{1}, P_{2}$, and $P_{0}$ will determine which of the three values will be selected for a given run. This procedure has been used to evaluate the COAL 2 National Energy Model, and to the author's knowledge, has not been used except in this one instance. The source for this description is Ford, Moore, and McKay [25]. 4.2.3.1 Evaluation The three-point sampling plan is actually only a special case of stochastic, factorial, stratified-sampling methodology. In this technique each input variable is assigned a density function, say $f_{1}(x)$, for $x_{i}{ }^{L}<x<x_{i}$. Values of $x$ in this range are defined such that the following relation is obtained:

$$
x_{i}^{L}=a_{i o}<a_{i l}<\cdots<a_{i k}=x_{i}^{H}
$$

and the probability of the interval $\left(a_{i, j-1}, a_{1, j}\right)$ is $1 / k$. These intervals, one for each input variable, are sampled to produce different values of input variables for each computer run.

There are two major objections to this sampling method. The first is the practical difficulty of assigning a density function for each input variable, particularly for a model with a large input data base. The second objection arises in the possible re-selection or assignment of values which may have been used in a prior run. This is the problem of sampling with replacement. This problem, along with the realization that the sampling procedure may not, 
based on probability selection, provide a proper spread of values which are needed to evaluate the model performance, creates the possibility that some degree of monitoring of values assigned is necessary and also that the number of needed runs may actually be increased beyond initial expectations.

The next Section will discuss how these difficulties are precluded in the Latin Hypercube Sampling method.

4.2.3.2 Application This scheme of sampling is for statistically independent input variables and models for which the preparation of the scheme is not excessively costly in time and resources. The number of runs for this type of statistical analysis is of the form

$$
\mathrm{N}=\mathrm{k}^{\mathrm{I}},
$$

where $k$ is the number of intervals and $I$ the number of input variables. For some models, those of long running times, a large $\mathrm{N}$ may prohibit the use of this approach.

\subsubsection{Latin Hypercube Sampling Method}

The principal references on this method are McKay, Conover, and Whitman [22], and McKay, Beckman, and Conover [23].

When using the stratifed sampling methods there are many options on the choice of the number of intervals ( $k$ ) of each input variable and the fraction of an experimental design wherein only some of the combinations of interest are actually evaluated. The Latin Hypercube Sampling (LHS) method uses the same number of intervals for each input variable as the number of computer runs to be made. The intervals for each input are assigned by defining a sequence of independent, uniform random variables, ranking them from lowest to 
highest, and then use this ranking to determine the computer run in which that input value will be used. As in the stratified sampling method the intervals must be sampled to obtain specific values of the input variables. The values, however, may be fixed by the model user instead of having the assignment dependent on an interval density function. This choice is an important option in terms of cost and preparation. This method can be regarded as an extension of Latin Square sampling.

Reference [23] demonstrates that the variance of an estimator based on LHS, in terms of the variance based on random sampling, is:

$$
V\left(T_{L}\right)=V\left(T_{R}\right)+\frac{(N-1)}{N}\left(N^{-k}(N-1)^{-k}\right) \sum\left(\mu_{i}-\tau\right)\left(\mu_{j}-\tau\right)
$$

where $\sum$ is over the restricted space of pairs $\left(\mu_{i}, \mu_{j}\right)$ having no coordinates

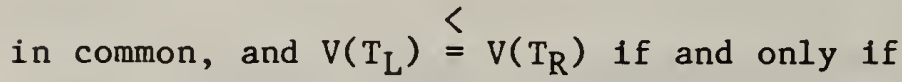

$$
N^{-k}(N-1)^{-k} \sum\left(\mu_{i}-\tau\right)\left(\mu_{j}-\tau\right) \stackrel{\leq}{=} 0
$$

No direct way of comparing the variance of an estimator based on the LHS to that based on stratified sampling is available. However Reference [23] states, and proves the assertion, that if a function $Y=h\left(x_{1}, \cdots, x_{k}\right)$ is monotonic in each of its arguments, and $g(Y)$ (see equation (4.4)) is a monotonic function of $\mathrm{Y}$, then

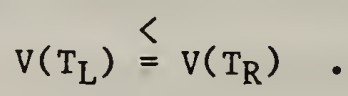

4.2.4.1 Evaluation The principal advantage of LHS is to overcome the difficulties of the stratified sampling method by insuring a representative coverage of all input values within the set of prescribed runs. The output of 
the sampling design uses all of the intervals on the range of each input variable. The proofs and mathematical developments given in References [22] and [23] assume a random selection, without replacement, for an input value.

It appears, however, that a predetermined assignment of values from a given partition on each input interval could be made which would allow a direct study of particular effects associated with certain values of the input data. This technique uses LHS as a probe for special-case examinations, by avoiding the necessity to extract such information from a more general statistical design. This application fixes the number of runs, however, and a tradeoff between the number of runs (or model evaluations) and the interval partition must be evaluated. To assign values might violate statistical requirements, but it would provide limited but important information on model performance.

The advantage of the LHS is revealed when the model output is dominated by only a few input variables. But the LHS ensures that each component is fully stratified, no matter which variables may or may not dominate the results.

In Reference [22] McKay, et al, conclude that factorial stratified sampling (FSS) and Latin hypercube sampling are clearly superior to estimates based on random sampling. The amount of gain, however, depends on the model under assessment, but in their experience the LHS resulted in a maximum variance reduction of about 50 to 1 over random sampling. Early studies also indicate LHS is "far superior" to FSS.

4.3 Analysis of Range

In a basic paper Tsukibayashi [26] has developed the moments of the range for rectangular, triangular, exponential, gamma, and normal distributions 
His results include a calculation of certain coefficients to be used in estimating the first four moments of the distribution.

Tsukibayashi states that the moments of the range are functions only of the sample size $N$ and the variance $\sigma$; the following are derived. If $W_{N}$ is the range, then:

$$
\begin{aligned}
& \mu_{1}=E\left(W_{N}\right)=d_{N} \sigma \text {, and } \\
& \mu_{2}=E\left(W_{N}^{2}\right)=C_{N}^{2} \sigma^{2} ;
\end{aligned}
$$

higher moments are not given here. The table below summarizes the results of

\begin{tabular}{|c|c|c|}
\hline Distribution & $\mathrm{d}_{\mathrm{N}}$ & $\mathrm{C}_{\mathrm{N}^{2}}$ \\
\hline Rectangular, $f(X)=1,0<x<1, \sigma^{2}=1 / 12$ & $12\left(\frac{n-1}{n+1}\right)$ & $12 \sqrt{\frac{n(n-1)}{(n+1)(n+2)}}$ \\
\hline $\begin{array}{l}\text { Triangular, } f(X)=2 X, 0<X<1, \sigma^{2}=1 / 18 \\
\text { ( } B \text { is the beta function) }\end{array}$ & $18\left[\frac{2 n}{2 n+1}-\frac{1}{2} B(1 / 2, n+1)\right]$ & $18\left[\begin{array}{c}\frac{1-2 n+1}{2(n+1)} \\
B(1 / 2, n+1)\end{array}\right]$ \\
\hline Exponential $, f(X)=e^{-x}, 0<x<\infty, \sigma^{2}=1$ & $\sum_{k=1}^{n-1} \frac{1}{k}$ & $\mathrm{~d}_{\mathrm{N}}^{2}+\sum_{\mathrm{k}=1}^{\mathrm{n}-1} \frac{1}{\mathrm{k}^{2}}$ \\
\hline
\end{tabular}
the analysis of range in terms of statistical parameters.

For the more complicated expressions for the gamma and normal distributtons of the range the reader should refer to [26], pages 66 and 67 . The theory 1 underlying the coefficients given above was developed by Tippett and Pearson. 4.4 Minimum Variance Sampling

For models which simulate the states of a system or activity, such as Markov processes, queues, or stochastic processes, the sampling is usually performed in proportion to their natural freauency of occurrence. A technique has been developed by Bayes [27] which allows the sampling frequency of the

1 The reference to Tippett is Riometrika, 17 (1925). 
states to be independent of their natural frequency. A sampling theory of Markov chains is given by Bayes which allows some statistics of the state frequencies to be estimated with minimum variance for a given sample size.

In models of this kind some states are experienced infrequently, which requires a large number of runs to collect enough data on these states to perform a proper evaluation. This situation has led to the development of "importance sampling" in which the simulation is constrained to experience rare states independently of the expected frequency of the number of times these states are actually realized in a normal use of the simulation.

Bayes applies the theory by approximating the model, in this case a queueing problem within a teleprocess simulation, by a Markov chain in which states are assigned, transition probabilities are given, and means and variances of intervals spent in each state are assigned. The statistical properties of the Markov chain surrogate model so defined will be similar to those of the original simulation.

\subsection{Regression}

The subjects of linear and nonlinear regression, and polynomial curve fitting are so familiar that the structures of these techniques will not be repeated here. This Section is included for the purpose of reviewing the contributions of regression to the sensitivity analysis of models.

A proper regression is known to reduce the length of a confidence interval and to influence the value of the model output, assuming a causal relationship. The regression model itself contributes information on sensitivity through the magnitudes of the coefficients and the numbers of 
variables taken into the regression formulation. Both curve-fitting and regression provide functional relationships which can be used according to the theory, given above, on error propagation and analysis.

We quote an important comment from Deming [13] on the matter of indirect contributions to knowledge based on regression analysis: "There have been many instances when deductions made from a fitted curve, or from a series of curves, have made it unnecessary to perform certain other experiments. As an instance ... where a quartic was fitted to compressibility data on carbon dioxide ... this quartic gave data on the index of refraction, the Joule-Thomson coefficient, entropy, and other physical properties that would be difficult and time consuming if direct observations were required." 4.6 Partial Rank Correlation Coefficient (PRCC)

McKay, et al [22], have proposed and used the PRCC as an alternative to the derivative approach. The partial correlation coefficient, originally employed to measure linear relationships among variables, is adjusted to use ranked data, and allows for nonparametric tests. Following [22] the formulation is as follows:

Let $\left(x_{1 i}, x_{2 i}, \ldots, x_{k i}, y_{i}\right)$ denote the values of $k$ input variables and the output variable for the ith computer run, $1=1, \ldots, n$. Let $\left(x^{r} 1 i, x^{r}{ }_{2 i}, \ldots, x_{k i}, y^{r}\right)$ denote the associated rank variables. For example, if $n=3$, and $x_{11}=12, x_{12}=25, x_{13}=10$, then $x_{11}^{r}=2, x_{12}^{r}=3$, and $x^{r} 13=1$

Let $C$ be a $(k+1) x(k+1)$ symmetric matrix of elements $c_{i j}$, when 
$c_{i j}=\frac{\sum_{t=1}^{n}\left(x^{r}{ }_{i t}-\bar{x}_{i}\right)\left(x^{r}{ }_{j t}-\bar{x}_{j}\right)}{\left[\sum_{t=1}^{n}\left(x^{r}{ }_{i t}-\bar{x}_{i}\right)^{2} \sum_{s=1}^{n}\left(x^{r} j \bar{s}_{j} \bar{x}_{j}\right)^{2}\right]^{1 / 2}}$

for $i, j=1,2, \ldots, k$, and

$c_{i, k+1}=\frac{\sum_{t=1}^{n}\left(x^{r} t^{-\bar{x}^{r}}{ }_{i}\right)\left(y^{r} \bar{y}^{r}\right)}{\left[\sum_{t=1}^{n}\left(x^{r}{ }_{i t}-\bar{x}_{i}\right)^{2} \sum_{s=1}^{n}\left(y^{r} \bar{s}^{-} r\right)^{2}\right]^{1 / 2}}$,

where $c_{i i}=1$ and $c_{i j}=c_{j i}$ for $i, j=1, \ldots, k+1$.

The values $\overline{\mathrm{x}}^{\mathrm{r}} i$ and $\overline{\mathrm{y}}^{\mathrm{r}}$ are:

$\bar{x}_{i}=\frac{1}{n} \sum_{t=1}^{n} x_{i t} r_{i t}=\frac{n+1}{2}, \bar{y}^{r}=\frac{1}{n} \sum_{t=1}^{n} y^{r}=\frac{n+1}{2}$.

Let $B=\left[b_{i j}\right]=C^{-1}$ then the PRCC between input variable $i$ and output variable $y$ is given by:

$$
r P_{i y}=-b_{i, k+1}\left(b_{i i} b_{k+1, k+1}\right)^{-1 / 2}
$$

The principal reference given in [22] is Steel [29] for the underlying theory for this approach. References [28] and [30] provide detailed accounts of the statistical theory of partial correlation coefficients. Reference [30] describes the theory from the perspective of geometric constructions in which all the relations are expressed in terms of lengths of vectors and 
subtended angles, thereby portraying the theory of partial correlation with the trigonometry of an n-dimensional constellation of points. 


\subsection{ANALYTICAL METHODS OF SENSITIVITY ANALYSIS}

\subsection{Introduction}

This section describes methods of sensitivity analysis which are analytical formulations rather than statistical designs, and are based on special needs that traditional methodology could not meet. In some cases the methods are experimental and of a singular nature, in others of a more solid nature which advance the state of the art through the introduction of innovative techniques or by the further development of established sensitivity analysis.

\subsection{Criterion Sensitivity Analysis}

\section{2 .1 Descripton}

This method has developed at the MIT Energy Laboratory, Model Assessment Group. The single source is Schwippe and Grubl [31], and seems not to have had much of a life after its genesis in the mid-1970's. In [31] the authors describe the methods as follow:

The model is be represented as $y=f(x)$, where $x$ is a vector of exogenous input parameters and $\mathrm{y}$ is a vector of model outputs. The perturbed output is $y(\Delta x)=f(x+\Delta x)$, where $\Delta x$ is the perturbation, $y(\Delta x)$ is the perturbed output, and $\delta y(\Delta x)=y(\Delta x)-y(x)$ is the output perturbation. Assume that some particular scalar criterion function $C$ of the output perturbation

$$
C(\Delta x)=C[\delta y(\Delta x)]
$$

has been selected. Then the basic idea of the criterion sensitivity analysis is to find that $\Delta x$ which maximizes $C(\Delta x)$. This can be viewed as a "worst case" analysis which finds the maximum sensitivity as measured by $C(\Delta x)$. The MIT approach was to choose $C(\Delta x)$ from one of the perturbed outputs $\delta y(\Delta x)$. 
The authors emphasize the usefulness of this method for models of a highly nonlinear nature.

\subsubsection{Evaluation}

This method was developed because simple linearization is not appropriate for many models. Criterion sensitivity analysis addresses in particular the problem of nonlinearity in the function-model represented by equation (5.1). An objection noticed by the authors is the difficulty in characterizing the input uncertainties (i.e. defining the space of $\Delta \mathrm{X}$ or its probability density). The advantage of the method is that it provides explicit mechanics for dealing with input uncertainties in a systematic fashion. No further descriptions of this method were available beyond [31] at the time this survey was prepared.

\subsection{Describing Function Sensitivity Analysis}

\subsubsection{Description}

Our source for the description of this technique is the same as for the criterion method, Reference [31], which is the basis for the following description:

With the describing function approach, the input perturbation vector, $\Delta x$, is taken as a random vector characterized by its probabilitv density $p(\Delta x)$. Any density function is permitted and it is not assumed that the members of $\Delta x$ are independent.

The describing function is defined to be a vector polynomial function of the vector $\Delta x$, as follows

$$
D(\Delta x)=A_{0}+A_{1} \Delta x+\sum_{m=1}^{M} e_{m} \Delta x t^{t} A_{2 m} \Delta x+\ldots
$$


where $M$ is the dimension and $e_{m}$ is a unit column vector (all zeros except 1 in the mth row). Equation (5.2) is simplifed to

$$
D(\Delta x)=A \phi(\Delta x)
$$

where $\phi(\Delta x)$ is a vector of " 1 "s, $\Delta x$, and powers and cross products of $\Delta x$ up to the number of terms desired, of dimension $\mathrm{K}$, and $\mathrm{A}$ is a matrix of $A_{0}, A_{1}$, etc.

The describing function problem is then defined as follows: Find values of matrix $A$ and the number of $K$ terms such that

$$
\delta y(\Delta x) \approx D(\Delta x)
$$

Without reproducing the remaining mathematical development the following concluding paragraph is cited [31, page 506] to indicate the detail and intent of this approach.

"In summary, the preceding process involves fitting a set of describing functions to a set of model runs that represent a set of points on the model's input-output response surface. The suggested manner of determining the best fit has been a weighted least squares approach, where the importances of the different points are weighted by the likelihood that the response is going to be in the neighborhood of those points. With a large enough set of describing functions, that is as $\mathrm{K}$ approaches $\mathrm{N}^{1}$, the fitting of the different points can be "perfect." Such "perfect" fits are highly susceptible to capitalization on chance effects, and the preceding discussions suggests that one way of avoiding such spurious fits is to restrict the number of describing functions

$1_{\mathrm{N}}$ is the number of perturbations, taken larger than $\mathrm{K}$. 
in the set being used. There are endless variations on this particular suggested procedure for determining the "best" fit. A few of these variations include:

(1) minimizing the maximum residuals between the surface and the points,

(2) minimizing absolute differences or some other robust measure rather than least squares, or

(3) using weighted maximum residuals or weighted robust techniques. In addition it would be possible to exploit any intuitions one might have about the true characteristics of the model's response surface. For example, if a large number of points were available and one's intuition suggested that the response surface should be a relatively smooth connection of those points, then the fitting criterion might be to minimize the integral of the deviation between the fitted surface and the piecewise linear connection of the available points with their nearest neighbors (or the supporting hyperplane n-gons connected over all convex sets of available points). The principal drawback to these more elegant techniques is that they may not be nearly as easily solved as is the least squares approach."

\subsubsection{Evaluation}

In addition to difficulties of establishing the character of the input uncertainties and the final comment of the cited paragraph given above, the authors assert that describing functions can be very useful in cases where the direction and magnitude of the changes in outputs is of greater interest than the relative effects of different types of hypothesized inputs. 


\subsection{Adjoint Sensitivity Analysis}

\subsubsection{Description}

A recent and important method of sensitivity analysis has emerged from the collective work of scientists and model builders at the Oak Ridge National Laboratory (ORNL). This method is called adjoint sensitivity analysis and had its origin in some earlier papers by Oblow [33,34] and Cacuc1 [36] which dealt with general sensitivity analysis of problems in nuclear cross-section, shielding, and reactor physics.

The theoretical method was recognized for its potential as a practical tool for evaluating the large economic - energy models which were also in use at ORNL.

The basic paper from which our description is taken is Alsmiller, et al, [32]. It provides a clear statement of the essentials of the method. The underlying theory and mathematics are given in [33-37]. Reference [38] describes the application of the method to an ORNL model called LEAP.

The following text, which describes adjoint sensitivity theory, is taken, with minor editing for clarity, from Alsmiller [32], pages 3-7.

Let the system under consideration be represented by $\mathrm{N}$ nonlinear algebraic equations in $\mathrm{N}$ unknowns which may be written symbolically as

$$
F(p, x)=0
$$

where $\mathrm{F}$ is an $\mathrm{N}$-vector function of the unknown dependent variable $\mathrm{N}$-vector $\mathrm{p}$ and all data elements are represented by the vector $x$. It is to be understood that the number of elements in $\mathrm{x}$ is not at all related to $\mathrm{N}$ and can be substantially larger. In the following it is assumed that for a specific choice of $\mathrm{x}$ a unique solution of Eq. (II.1) exists and is represented by $\mathrm{p}$. The vector $p$ is thus a function of $x$. 
Let $R$ represent any result, hereinafter also called a response, of the calculations that are of interest. In particular, let

$R \equiv R(p, x)$,

wehre $R(p, x)$ is any given nonlinear function of $p$ and $x$. The response $R$ is a scalar that may be calculated from Fa. (II.2) when the solution p of Fa.

(II.1) has been obtained for a given specification of $x$.

If now $x$ is defined to be a general element of $x$ the sensitivity of $R$ to $x$ is defined to be $\frac{d R}{d x}$ and the general problem of sensitivity theory is to determine $\frac{d R}{d x}$ for each and every one of the elements of $x$. To accomplish this, differentiate Eq. (II.2) with respect to $x$, to obtain:

$$
\frac{d R}{d x}=\frac{\partial R}{\partial x}+\frac{\partial R}{\partial p} \bullet \frac{d p}{d x}=0
$$

where $\frac{\partial R}{\partial p}$ is a row vector. The first term on the right-hand side of of $F q$. (II.3) is called the "direct effect" of $x$ on $R$ and the second term on the right-hand side in Eq. (II.3) is called the "indirect effect" of $x$ on $R$, since it reflects the implicit effect of $x$ on $R$ through $p$.

The evaluation of the second term on the right-hand side of Eq. (II.3) begins with the differentiation of Eq. (II.1) with respect to $x$ to give

$$
\frac{\partial F}{\partial x}+\frac{\partial F}{\partial p} \cdot \frac{d p}{d x}=0
$$

Now defining two new $\mathrm{N}$-vectors 


$$
\begin{aligned}
& \Phi \equiv \frac{d p}{d x} \\
& S \equiv-\frac{\partial F}{\partial x},
\end{aligned}
$$

and the $\mathrm{N}$ by $\mathrm{N}$ matrix

$$
A \equiv \frac{\partial F}{\partial p},
$$

Eq. (II.4) may be rewritten as

$$
\mathrm{A} \Phi=\mathrm{S}
$$

Here $\Phi$ is the differential change in the solution $p$ with respect to a change in $\mathrm{x}$ and it solves the linear inhomogeneous equation whose source is the negative of the partial differential change in $F$ with respect to $x$. The fact that $\mathrm{A}$ is a linear operator is clear from Eq. (II.4) since it cannot, in principle, depend on $\frac{d p}{d x}$. The matrix A does depend on both $p$ and $x$.

The expression for $\frac{\mathrm{dR}}{\mathrm{dx}}$ in Eq. (II.3) can now be evaluated by solving Eq. (II.8) for $\Phi$. The difficuly here lies in the fact that $S$ depends explicitly on the particular $\mathrm{dx}$ being considered and thus, in general, very large systems of linear equations represented by Eq. (II.8) must be solved for each $\mathrm{dx}$ to obtain $\frac{\mathrm{d} R}{\mathrm{dx}}$. For very large systems where $\mathrm{x}$ contains thousands of elements this is impractical. It should be noted that the explicit assumption is being made here that it is not feasible to numerically construct the inverse of the matrix A. If this inverse could be obtained then the need for solving the 
large systems of linear equations many times would be ameliorated and the above objection would not be valid.

The adjoint formulation of sensitivity theory is of interest hecause it avoids the necessity of solving a large set of linear equations for every $x$ in $x$. The set of equations adjoint to the Eq. (II.8) will be written

$$
A^{*} \Phi^{*}=S^{*}
$$

where the $\mathrm{N}$ by $\mathrm{N}$ matrix $\mathrm{A}^{*}$ is defined ${ }^{l}$ to be:

$$
A^{*}=A^{t}
$$

where a superscript * is used to denote adjoint, a " $t$ " over a symbol is used to indicate a transpose, and where $S^{*}$ is yet to be defined. If now the inner products of $\phi t$ with Eq. (II.9) and $\Phi * t$ with Eq. (II.8) are made one obtains, by using Eq. (II.10):

$$
\Phi t \bullet S^{\star} t=\Phi^{*} t \bullet S
$$

and it $S^{*}$ is defined ${ }^{2}$ by:

$$
S^{*}=\frac{\partial R^{t}}{\partial p}
$$

then using Eqs. (II.11) and (II.12), Eq. (II.3) may be written

$$
\frac{d R}{d x}=\frac{\partial R}{\partial x}+\Phi^{* t} \bullet S
$$

where $\Phi^{*}$ is the solution of Eq. (II.9), with $S^{*}$ given by Eq. (II.12).

${ }^{1}$ The proof of this definition is given in Cacuci, et al, [36]. The matrix A* is formed by transposing the adjoints of the components of $A$.

${ }^{2}$ The justification for this definition is given in Cacuci, et al, [36] 
Equation (II.13) is the important result of adjoint sensitivity theory.1 The quantity $\Phi^{*}$ must be obtained by solving the, often large, set of linear equations given in Eq. (II.9), but this $\Phi^{*}$ may be used in Eq. (II.13) for $\mathrm{x}$ of the vector $\mathrm{x}$. Thus, one calculation of $\Phi^{*}$ enables the calculation of $\frac{d R}{d x}$ for all $x$ in $x$ for a specific $R$. Each new response, $R$, that is considered requires a new calculation $\Phi^{*}$ because of Eq. (II.12).

\subsubsection{Evaluation}

The observations on the effectiveness and efficiency of adjoint sensitivity analysis are taken from Alsmiller [38], with some minor emendations and omissions.

Several important observations should be made in regard to the results. The first advantage of this approach is that the adjoint equation is independent of any operation involving differentiation with respect to $x$. This property means that, no matter how large the set of input parameters is, only a single adjoint equation needs to be solved to compute $\mathrm{dR} / \mathrm{dx}$ for all $\mathrm{x}$ in $\mathrm{x}$.

This is in contrast to any direct method of computing $d R / d x$ by changing each $\mathrm{x}$ by a finite amount (i.e., $\Delta \mathrm{x}$ ) and solving Eq. (II.1) to approximately get $\mathrm{dR} / \mathrm{dx} \cong \Delta \mathrm{R} / \Delta \mathrm{x}$ or by using $\mathrm{Eq}$. (II.8) directly to solve exactly for $\mathrm{dp} / \mathrm{dx}$. In both of these latter cases, the solution of a large system of equations [i.e., either Eq. (II.1) or II.8)] is needed for each parameter $x$ to be studied. The adjoint approach is therefore extremely economical to use, and

${ }^{1}$ An alternative derivation of equation II.13 is given in Alsmiller, Jr., et al., [53]. This derivation is based on an algebraic representation rather than on linear operator theory. 
with a very large set of input parameters, it may be the only approach to obtaining all sensitivity coefficients.

The second advantage is that the adjoint equation is a linear equation in $\phi^{\star}$, which is generally much easier to solve than the repeated calculations using Eq. (II.1), which is nonlinear. The third advantage is that this approach allows the exact sensitivity coefficient $\mathrm{dR} / \mathrm{dx}$ to be evaluated by using Eq. (II.13) (i.e., no perturbations in $\mathrm{x}$ and no approximations such as $\Delta \mathrm{K} / \Delta \mathrm{x} \cong \mathrm{dK} / \mathrm{dx}$ are needed).

On the other hand, certain limitations and disadvantages of this method are also apparent. The first and foremost disadvantage is that, to construct the adjoint equations [i.e., Eq. (II.9)] and evaluate dR/dx with Eq. (II.13), various derivatives of $f$ and $R$ must be evaluated analytically. This evaluation requires an in-depth knowledge of the functional form of the complete set of equations described by $\mathrm{f}$. Second, the structure of the $A^{*}$ matrix, although independent of $\phi^{*}$, is not independent of $p$. This means that the solution of Eq. (II.1) must be available to construct $\mathrm{A}^{*}$, thus tying the adjoint equations to the solution of the forward equations. Therefore, to evaluate Eq. (II.13) for $d R / d x$, both solution vectors $p$ and $\phi^{*}$ must be available in addition to all analytic derivatives of $f$ and $R$ with respect to both $\mathrm{p}$ and $\mathrm{x}$ in $\mathrm{x}$. A tradeoff study between analytic work in the adjoint approach and computational time in the alternative forward approaches may be necessary; the usefulness of either method will depend on the circumstances of the sensitivity study being contemplated. 
One additional disadvantage of the adjoint approach is that an adjoint solution is required for each new response whose sensitivity is being studied. This is apparent from the source term in Eq. (II.9), which depends on the definition of the response $\mathrm{R}$. This highlights the complementary nature of forward and adjoint methods. The forward approach gives the sensitivity of all responses to one parameter in one run, whereas the adjoint method gives the sensitivities of one response to all parameters in one run. From a sensitivity point of view, the adjoint method is thus clearly more applicable to problems with large numbers of input parameters and few responsesof-interest.

\subsubsection{Application}

A particularly striking example of the power of adjoint theory in the measurement of model sensitivity is given by oblow [34]. In this paper oblow applies adjoint theory to a linear program problem and to other nonlinear problems with and without given constraints. Oblow concludes that "the developments presented make it clear that [adjoint] sensitivity theory can be extended successfully to cover a wide class of algebraic nonlinear equations with and without constraints." He continues, "the sensitivity coefficients made available by the methods developed here can be put to a number of uses. For example, Taylor series expansions using sensitivity coefficients can be used as the basis for a second-order accurate perturbation theory for the nonlinear systems under investigation. In addition, a statistical uncertainty analysis of system responses can also be made if perturbation results are combined with assumptions about the nature of the uncertainties in the system input parameters". 


\subsection{Linear Programming}

\subsubsection{Description}

\subsubsection{Postoptimality Evaluation The method of linear programming is so}

well known that this section will discuss its contribution to sensitivity analysis methodology with only a reminder that linear programming entails the optimal solution of an algebraic formulation of a problem expressed as a linear objective function subject to linear constraints. Its connection to sensitivity analysis is the postoptimality evaluation of a solved linear program.

Following Taha [39], the changes in the problem include variations of: (1) the right-hand side of the constraints (resource vector), (2) the coefficients of the objective function (cost or profit values), (3) the coefficients of decision variables (matrix of technological coefficients); or the addition of: (5) new variables, and (6) new or secondary constraints. The sensitivity associated with these changes is determined by the affect they have on the basic variables of the optimal solution: the basic variables may remain essentially unchanged; the basic variables remain but their values change; or the basic solution changes completely. The extent to which these variations can be made without changing the basic variables of the solution, or essentially changing the value of the objective function, constitutes a sensitivity analysis of the coefficients and constraints of a linear program model. A statement of the primal and dual formulations of a linear program are given in Appendix B.

\subsubsection{Parametric Sensitivity A related form of sensitivity analysis is} called parametric linear programming. It investigates the response of the solution to predetermined linear variations in the coefficients and resource vector based on the primal-dual relationships used in the postoptimality 
formulation [see [39], chapter 9]. Further details on parametric programming are given in Appendix B. There the various forms an analysis may take are presented and a general review of the analytic procedure is provided. Parametric linear programming is a well known technique of sensitivity analysis and has been incorporated into linear programming software packages as a standard operating procedure for linear program problems. 5.5.1.3 Tolerance Sensitivity of considerable interest and as a innovative contribution to linear programming sensitivity analysis is the recent work of Wendel1 [40] on the subject of tolerance evaluation in a jinear program problem. His abstract summarizes his contribution: "In contrast to 'ordinary' sensitivity analysis in linear programming, the tolerance approach considers simultaneous and independent changes in the objective function coefficients and in the right-hand-side terms. This approach calculates a maximum tolerance percentage such that as long as selected coefficients or terms are accurate to within that percentage of their estimated values, the same basis is optimal. In particular, if the objective function coefficients are accurate to within the maximum tolerance percentage of their specjfied values, then the same solution is optimal." Wendell states in his introduction that his tolerance approach is a new perspective that permits a sensitivity analysis which deals with more than one coefficient term at a time and yields the largest percentage in which terms may vary simultaneously and independently from their numerical values while retaining the same optimal basis. His method also yields an analogous result for the coefficients of the objective function.

5.5.1.4 Lagrangian Parametric Sensitivity A method of considerable importance for the study of parametric sensitivity has been developed from the 
classical Lagrangian optimization technique. In the economic literature the method is referred to as the envelope theorem, but its derivation and use in general analytic form makes it universally applicable to any constrained or unconstrained objective function. The theorem provides an equation for the computation of the rate of variation of an objective function with respect to variation in a specified parameter of interest. The following derivation follows Silberberg [54].

Maximize an unconstrained function $U$, defined as: $\mathrm{U}=\mathrm{f}\left(\mathrm{x}_{1}, \ldots, \mathrm{x}_{\mathrm{m}} ; \alpha\right)$

From the Lagrangian technique for the optimum value of $U$, $U^{\star}$, we write $U^{*}=\phi(\alpha)=f\left(x_{1} *(\alpha), \ldots, x_{n}^{*}(\alpha)\right)$, where $x_{1}^{*}(\alpha)$ are the optimal values of $x_{i}$ as functions of the parameter $\alpha$. The sensitivity coefficient is, therefore:

$$
\frac{\partial U^{*}}{\partial \alpha}=\frac{\partial \phi}{\partial \alpha}=\frac{\partial f}{\partial \alpha}
$$

If now the same problem is solved using the Lagrangian technique under the constraint functions $g_{j}$, i.e.:

$$
g_{j}\left(x_{1}, \ldots, x_{n} ; \alpha\right)=0 \text {, for } j=1, \ldots, m \text {, then }
$$

The sensitivity coefficient becomes:

$$
\frac{\partial U^{*}}{\partial \alpha}=\frac{\partial \phi}{\partial \alpha}=\sum_{i=1}^{n} \frac{\partial \mathrm{f}}{\partial \mathrm{x}_{i}^{\star}} \frac{\partial \mathrm{x}_{i}^{*}}{\partial \alpha}+\frac{\partial \mathrm{f}}{\partial \alpha}
$$

Differentiating each constraint function with respect to the parameter $\alpha$, we obtain:

$$
\sum_{i=1}^{n} \frac{\partial g_{j}}{\partial x_{i} *} \frac{\partial x_{i *}}{\partial \alpha}+\frac{\partial g_{j}}{\partial \alpha}=0, \text { for each } j=1, \ldots, m
$$


then multiplying by $\lambda j$ and adding the equations to $\frac{\partial \phi}{\partial \alpha}$.

The equation of the envelope theorem follows, and is given by:

$$
\frac{\partial u^{*}}{\partial \alpha}=\frac{\partial L}{\partial \alpha}=\frac{\partial f}{\partial \alpha}+\sum_{j=1}^{m} \lambda_{j} \frac{\partial g_{j}}{\partial \alpha}
$$

where $L$ is the Lagrangian function, $f+\sum_{j}^{m} \lambda_{j} g_{j}$.

This method for computing the sensitivity coefficients for objective functions suffers from the same computational difficulties associated with the Lagrangian optimization technique, primarily because the optimal values and the multipliers may be tedious to obtain for complex or large problem formulations.

However, the method is attractive because it computes the sensitivity of objective functions with respect to any parameter in the formulation; that is the parameter may be a resource level, a cost or profit coefficient, or a technical coefficient. Since the objective function and constraint functions may be nonlinear the envelope theorem permits the concept of linear parametric sensitivity analysis to be carried over into the the domain of the parametric sensitivity of nonlinear, optimization problems.

\subsection{Sensitivity Functions}

\subsubsection{Description}

The sensitivity function is derived from a differential equation

$$
F(x, x, x, t, q)=0
$$

in which $\mathrm{q}$ is a parameter to be perturbed. If we assume a solution to equation $(5.6), x=x(t, q)$, we next determine the effect on $x$ of a perturbation, $\Delta q$, in $q$. This gives

$$
F(x, x, x, t, q+\Delta q)=0
$$


as the new system differential equation. We now define a variable, termed the sensitivity coefficient, as $u(t, q)$ which is the derivative of $x$ with respect to $\mathrm{q}:$

$$
u(t, q)=\lim _{\Delta q \rightarrow 0} \frac{x(t, q+\Delta q)-x(t, q)}{\Delta q}=\frac{d x(t, q)}{d q}
$$

The sensitivity coefficient measures the change in $x$ due to a pertrubation in q. Differentiating equation (5.6) with respect to $q$ gives:

$$
\frac{\partial F}{\partial \ddot{x}} \frac{\partial \ddot{x}}{\partial q}+\frac{\partial F}{\partial \dot{x}} \frac{\partial \dot{x}}{\partial q}+\frac{\partial F}{\partial x} \frac{\partial x}{\partial q}+\frac{\partial F}{\partial q}=0
$$

with $\frac{\partial \dot{x}}{\partial q}=\frac{\partial}{\partial t} \frac{\partial x}{\partial q}=\dot{u}$ and $\frac{\partial \ddot{x}}{\partial q}=\frac{\partial^{2}}{\partial t^{2}} \frac{\partial x}{\partial q}=\ddot{u}$;

then equation (5.8) takes the form

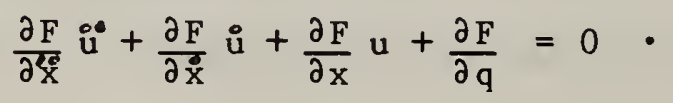

Equation (5.11) is the sensitivity equation which is to be solved for $u(t, q)$. The sensitivity equation is a linear equation, and provided the initial conditions for equations (5.6) and (5.7) are the same, all initial conditions of equation (5.11) are equal to zero. See References [41] and [42] for more detail on the theory and scope of the sensitivity function. Reference [43] demonstrates an application to a world dynamics model based on a system of nonlinear equations of the first order. 


\subsection{Eigenvalue Functions}

\subsubsection{Description}

$$
\begin{aligned}
& \text { In a system of linear first-order differential equations } \\
& \dot{y}=\text { Ay }
\end{aligned}
$$

where $y$ is an $n$-component state vector and $A$ an $n$ by $n$ matrix of coefficients, the solution is of the form

$$
y=c x e^{\lambda t}
$$

where $\mathrm{x}$ is an $\mathrm{n}$-component vector and $\mathrm{c}$ is an arbitrary scalar. When equation (5.13) is substituted into equation (5.12) the problem is reduced to finding the eigenvalues of the system of equations.

$$
A x=\lambda x
$$

If it is necessary to study the parametric effects of variations in the eigenvalues then equation $(5.14)$ is partially differentiated with respect to a parameter $q$ and the variational equation is obtained

$$
\frac{\partial A}{\partial q} x_{i}+A \frac{\partial x_{i}}{\partial q}=\lambda i \frac{\partial x_{i}}{\partial q}+\frac{\partial \lambda_{i}}{\partial q} x_{i}
$$

for each $i$, the index over the vector $x$. After some mathematical manipulation the sensitivity coefficient is expressed by

$$
\frac{\partial \lambda_{i}}{\partial q}=\frac{\left(\frac{\partial A}{\partial q} x_{i}, v_{i}\right)}{\left(x_{i}, v_{i}\right)}
$$

where the notation $(\cdot, \cdot)$ indicates a scalar product, and $v_{1}$ is the ith eigenvector of the system when the matrix A is transposed. For a given 
eigenvalue, $\lambda_{i}$, the eigenvectors $x_{i}$ and $v_{i}$ are calculated, then equation (5.15) is used to compute the sensitivity coefficient. This description is based on material contained in Reference [42].

\subsection{Large Error Sensitivity Analysis}

\subsubsection{Description}

By large error we mean those errors of sufficient magnitude which render the classical linear error equations invalid as measures of sensitivity. Classical error analysis assumes that variations behave with necessary properties, (1) that they are small, i.e., neighborhood variations, and (2) that on average there are as many negative variations as there are positive variations. This second assumption is critical to the classical formulation of variance.

The evaluation of sensitivity coefficients for large errors rests on using more complicated formulae where second order terms are not dropped, on using the variational form of the differential method described in Section 3.3 , or on the use of interval analysis in which extreme values of a function serve to delineate the range of a function under perturbation.

Rahman [44] gives several examples of simple operations such as $\mathrm{x} / \mathrm{y}$ where both variables are subject to variation. Letting $z=x / y$, the largest change in $x / y$, excluding $y=0$, will be when $x$ is at its largest value and $y$ at its smallest. Similarly the smallest value of $\mathrm{Z}$ will occur when $\mathrm{x}$ is minimized and $y$ takes its greatest variational value. This gives $z_{u}$ and $Z_{L}$ where

$$
\mathrm{Z}_{\mathrm{u}}=\min _{\mathrm{y} \max }[\mathrm{z}=\mathrm{x} / \mathrm{y}]
$$

and $\mathrm{Z}_{\mathrm{L}}=\max _{\mathrm{y}} \min _{\mathrm{x}}[\mathrm{Z}=\mathrm{x} / \mathrm{y}]$ 
or $\mathrm{Z}_{\mathrm{L}} \leqslant \mathrm{Z}<\mathrm{Z}_{\mathrm{u}}$. If anything is known about the distribution of $\mathrm{x}$ and $\mathrm{y}$ in the intervals of their variation then the theory described in Section 4.3 can be used to obtain estimates of statistical parameters for $Z$.

This concept can be generalized by estimating the absolute maximum and minimum values of a function over the permitted hypercube of variations of the independent variables. If the range of variation is large but the relative error $(i e . \delta x / x)$ is less than one then some simplifying substitutions may be possible in the determination of the maximum and minimum. If the function does not possess a workable probability distribution over the hypercube of permitted variation then the sensitivity can be expressed as an absolute error $( \pm \varepsilon)$, or as a percent error of this expected value of the function over the range of the dependent variable. 


\subsection{SPECIAL ANALYTICAL METHODS OF SENSITIVITY ANALYSIS}

\subsection{Introduction}

In this section three very specialized methods of sensitivity analysis are described. They are included for various reasons - the Fourier coefficients method has some interesting properties, geometric programming is a useful tool for studying engineering costs prior to project commitment, and Catastrophe Theory, much like Game Theory, provides a conceptual structure in which many problems of a diverse nature can be analyzed with greater insight.

\subsection{Fourier Coefficients}

\subsubsection{Description}

The principal source is Cukier, Levine, and Shuler [45] from which we abstract, with minor editing, a portion for the description of the method.

"Separate in definition from the independent variables and parameters are the fixed constants of a model, which do not vary within the context of the class of problems of interest to the model user, and whose values can be precisely specified. It should be noted, of course, that what is a fixed constant in the context of one situation might be a parameter in the context of another situation; the distinction depends on the particular case on hand. The fact that the parameters can take on a range of values suggests that a statistical approach to sensitivity analysis is appropriate. Instead of considering the effect on the output functions of one-at-a-time variations in each of the parameters, as in a "brute force" method, we will construct outputs averaged in one operation over probability distributions of all the 
parameters. The distribution of the parameters can arise because of experimental uncertainties or theoretical approximations, because of "ignorance" of the value within certain reasonable bounds, or might represent upper and lower limits due to "stops" on the physical controls of the systems being modeled.

"Our method of sensitivity analysis proceeds by relating the probability distribution of each parameter to a frequency and one new parameter $s$ which, as $s$ varies, carries all the parameters through their range of variation. The parameter $s$ is varied, and the frequencies are chosen in such a way that the output variables at any fixed time become periodic in $s$.

"The output variables can then be evaluated using Fourier Analysis. As we shall show below, the Fourier coefficients represent an average of the output variables over the uncertainties of all the parameters. A unique correspondence between the Fourier coefficients for the frequency $w_{1}$ and all its harmonics and the sensitivity of the output variables to the kth parameter is established. We compress all this information into partial variances $S_{w l}$ which are the normalized sums of the squares of the Fourier coefficients of the fundamental frequency $w_{1}$ and all its harmonics. If $S_{w 1}<S_{w i}$ for a given output variable, then this output variable is less sensitive to the kth parameter than to the jth parameter. Thus, the partial variances measure the average sensitivity of an output function to the variation (or uncertainty) of a particular parameter. This average is over the range of uncertainties of all the parameters, with their appropriate probability distributions, with the exception of the parameter being considered. For this parameter, the statistical property calculated is the variance. 
"The sensitivity analysis is nonlinear so that it permits us to examine large deviations from the nominal parameter values. In addition, since all parameters are varied simultaneously, one explores regions of parameter space where more than one parameter is far from its nominal value. Because of this thorough and systematic exploration of the parameter space, it of ten turns out that sensitivities of an unexpected nature are revealed. A careful study of the model will then reveal some complex coupling between variables, unexpected prior to the analysis, which leads to the observed sensitivity. In this fashion, one obtains deeper insights into the structure of the complex system being studied. Another frequent and important finding is that a number of senstivity coefficients corresponding to a large set of parameters turn out to be negligible. This permits one to reduce the complexity of the set of model equations and focus one's attention on a greatly reduced set of equations."

\subsection{Geometric Programming}

\subsubsection{Description}

Geometric programming, a well known method of optimization of nonlinear problems, gives the optimal cost or profit before a corresponding design or plan is implemented. The method is described in detail in Duffin, Peterson and Zener [46], and is given as excellent exposition in Wilde and Beightler [47].

Instead of obtaining an optimal solution of the decision variables initially, geometric programming finds the optimal distribution of the total cost among the terms of the objective function, and when these optimal assignments are obtained the optimal cost follows directly from a computation. Once the optimized cost and weights are determined, they can be 
used to determine the optimal policy. Geometric programming can be used to study the policy consequences of variations in cost and in engineering design variables.

\subsection{Catastrophe Theory}

\subsubsection{Description}

Catastrophe theory describes the phenomena associated with sudden changes of state in a system caused by smooth alterations in the control parameters of the system. The theory is not easy to master. It is derived from topology which is needed to describe forces as smooth surfaces of equilibrium, but it also is not, according to Poston and Steward [48], a single thread of ideas, but embraces geometry, algebra, singularity theory, physical intuition and experiment. The sources on this subject are References [48-52].

The underlying concept of catastrophe theory is the recognition of the various kinds (in a mathematical sense) of catastrophes and the relationship between the catastrophe manifold and the control parameter space.

The catastrophe manifold or equilibrium surface is a set of points $\left(x,\left\{a_{i}\right\}_{N}\right)$, where $x$ is a state variable and $\left\{a_{i}\right\}_{N}$ is a set of $N$ control variables, $a_{i}$. The map of $\left(x,\left\{a_{i}\right\}_{N}\right)$ will have one or more folds in its surface which are a function of the potential of the energy of the system. The projection of the equilibrium surface, $\left\{a_{i}\right\}_{N}$ or

$$
\left(x,\left\{a_{i}\right\}_{N}\right) \rightarrow\left\{a_{i}\right\}_{N}
$$

defines the control parameter space. The folds of the equilibrium surface map into cusps in the control parameter space. As long as the values of $\left\{a_{i}\right\}$ are outside the enclosed region of the cusps the state variable will experience 
smooth, stable transitions. When, however, the values of $\left\{a_{1}\right\}$ cross into the cusp region the state variable is immediately unstable and will jump to a higher or lower value when the $\left\{a_{i}\right\}$ path or trajectory exits from the cusp region. Throughout this process the path of the control parameters values is smooth and continuous.

This subject bears on both the qualitative and quantitative nature of sensitivity analysis. The qualitative aspect lies in the insistence the theory makes on the model builder to study his subject closely for unstable or jump conditions in the state variables of his system, and to include these possibilities in his model. Far too often models fail because the underlying process was assumed to be a steady-state system when in fact it was subject to "catastrophic" changes of state.

The quantitative aspect of catastrophe theory lies in its broad application to many diverse types of problems. It has been used in physics, biology, social behavior, species population analysis, medical problems and models of ecological phenomena. It is not necessary in all cases to use catastrophe theory to assess systemic instability. However, even in these cases the knowledge of its elements should be very helpful in understanding the phenomena of state transitions. There are several catastrophe "flags" which signal certain conditions. Some aspects of system dynamics and associated flags are discussed in Appendix $C$. An understanding of systemic flags enhances the quality of a model if the model builder anticipates the impact of these situations and incorporates their consequences in the model structure and dynamics.

Catastrophe theory can contribute to a quantitative sensitivity analysis by using its basic ideas to compare model results with historical results. 
This application is particularly relevant to forecast models, economic models and time-series analysis. A plot of the values of the control variables over time versus system state data will show the effects of the model performance in comparison to the system performance over the period of interest. This comparison should provide a revelation of detail that will indicate how and in what manner control parameters relate to the system state variables and to the simulation of the system. 


\subsection{MODELING AND SENSITIVITY ANALYSIS}

\subsection{Introduction}

In this final section various ideas will be detailed which bear on the need to bring sensitivity analysis into a closer and more substantive relationship with model development. Since most of the methods covered in this survey are external to the model they result in a large investment in time, in data preparation, in input selection, and in runs of the model. Some of these separately, and undoubtedly all of them together, either defeat or discourage sensitivity studies because of the burden of having to impose the sensitivity methodology on the model in an external and inefficient procedure.

Attempts will be made to suggest ways to alleviate some of these burdens of testing a model's sensitivity to input data uncertainty and control parameter variation.

\subsection{Modeling Control Parameters}

A parameter is a value of a constant that determines the character of behavior of something which is a function of that parameter. The value of the control parameter is determined at the discretion of the user of the model.

Most models have many control parameters embedded in their logic which are usually constants whose values are set initially as input data to the model. The parameter is then fixed at its given initial value for the entirety of the model run. Static models, for the most part, are adequate which use this procedure, but models that simulate systems that are dynamic or predictive are severely restricted by this approach. 
To address this problem, and as appropriate for the model in question, each important control parameter should be constructed as a time or indexdependent variable, with each value per time-period or index number given as input. This allows the decision maker to vary the control parameter according to the conditions to be examined and permits a more realistic simulation of exogenous events which impact on or influence system behavior.

Another version of this idea is to assign values to a control parameter vector and have the model access the appropriate value of the control parameter successively, according to an index or a prescribed, sequential assignment.

Making the control parameter a variable by one of the above techniques provides the user with a program procedure for studying dynamic changes in the model scenario. It also provides a method for perturbing the values of control variables, in a variational sense, that will generate data which can be used in a sensitivity analysis of the model's performance.

\subsection{Modeling Sensitivity Methodology}

In almost every scheme of current methodology for analyzing the sensitivity of a model the emphasis is either on appropriate generation of input data, defining response functions, or performing a postpartum statistical analysis in which the model serves as the experiment. Other methods analogize the model as an operator performing on input data to produce output which is then tested on the basis of linear sensitivity formulations. The model is, in these experiments, perceived and treated as a black box. The sensitivity analyses of this approach provides an overall measure but it does 
not describe the relational dependencies and sensitivities experienced within the components of the model, of which there may be many and of a complex nature.

In order to reduce some of these objections, and at only a reasonable increase in the initial investment, it is suggested that sensitivity analysis should be an integral part of a model, incorporated into its logic, and structured to provide information on validation. The general concept of this integration would be as follows.

A model, in its initial stages of development, would normally be divided into its major components, as most models are; but this partitioning would be extended, within the components, to groupings of self-contained computations, which we will call mathematical modules. Assuming, for simplicity, that the flow chart of a portion of the model is shown as in Figure 1.

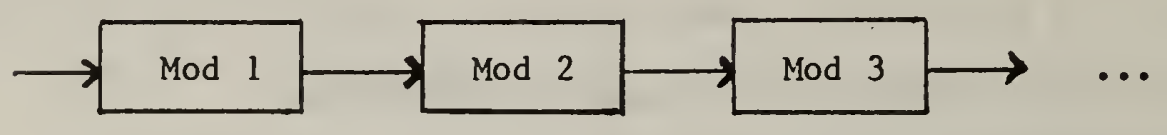

Figure 1

It is not important from this point on to retain the identity of the components since the design is based only on the modules. The scheme is now enhanced by providing each module with a program which provides a sensitivity subroutine appropriate for the computations performed by the module. This enhances the structure, as shown in Figure 2. 


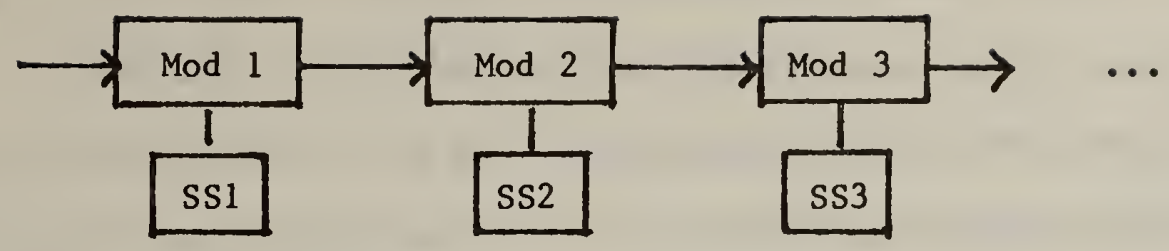

Figure 2

Next it is necessary to provide two operational paths through the flow chart of Figure 2. The path shown in Figure 3 is the normal operational use of the model without invoking the sensitivity subroutines (SS).

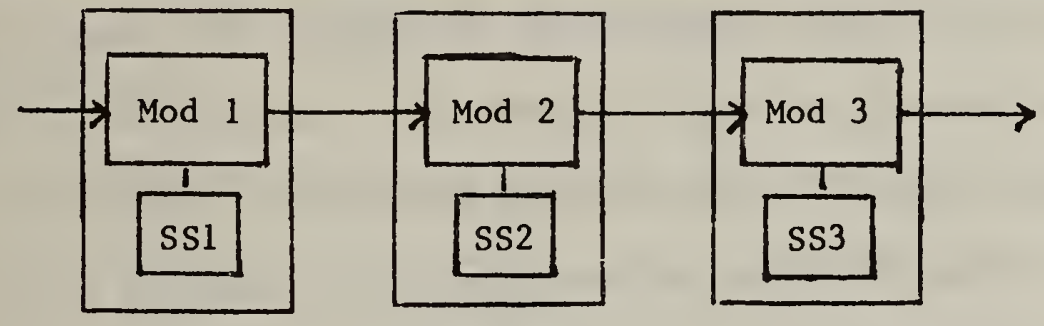

Figure 3

To obtain the second path it is necessary to construct a control vector, " $x$ ", composed of a sequence of logical control switches, one for each SS, which which will be "opened" or "closed" according to whether all or some of the sensitivity subroutines will be activated. If they are all used then the operational flow will be as in Figure 4.

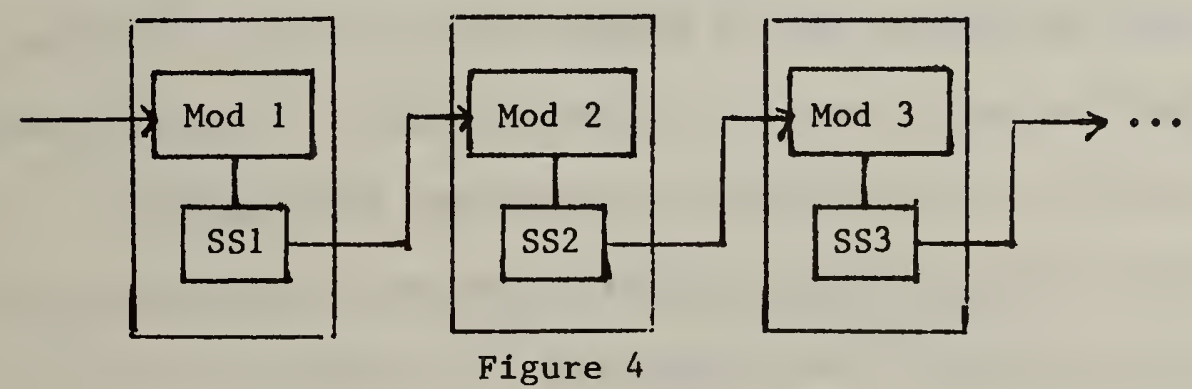

Figure 4 presents a basis for a number of variations on the essential concept. For example, if each senitivity subroutine can be optioned to output 
its calculations, then a running profile of the modular sensitivities can be obtained. The sensitivity subroutines could be written to pass their computations forward to the next SS operation, and so accumulate their results for some appropriate output option point. The information needed by each SS, primarily the variations, could be stored directly in an SS program, or some scheme could be programmed to have each activated SS compute its own variational values based on statistical parameters, relative errors, or functions.

The control variable $\mathrm{X}$ would be a vector of "on" or "off" signals which would be processed as the initial operation of the model. This vector would determine the process path of the model for that run. Another control vector could be used to signal to each SS how it is to compute the values of the variations it is to use in computing the sensitivity response of its associated mathematical module.

By whatever means it is accomplished, by the above concept or by inventions of new techniques, it appears that with the growing interest in model validation and sensitivity, it is essential to incorporate sensitivity methodology directly into the model structure.

The current practices of performing sensitivity analyses are not wholly satisfactory because of the assumptions which must be made and the often high cost of using a model as a black box in an experimental design from which the sensitivity analysis is carried out as a postpartum exercise. Incorporating the sensitivity analysis into the model logic provides more detail to validation and sensitivities studies, and offers the user a procedure for assessing the structural details and dynamics of the system and its simulation. 


\section{Appendix A}

Part I: Summary of Principal Error Formulas.

The principal formulas associated with the propagation of errors are presented for convenience and for the purposes of handy reference. These formulas are given in functional form in order to show their character and structure, and to generalize the formulas to multi-dimensional representations.

In the formulas the following definitions, symbols, and notations will be used:

$\delta \mathrm{x}$ is used to indicate a variation in a prescribed variable, here designated as $\mathrm{x}$;

$F$ is used to indicate a function; $F\left(x_{i}\right)$ is used to indicate a function of a set of variables $\left\{x_{1}\right\}$, with the index 1 running from 1 to $k$;

$r_{x_{i}} x_{S}$ is the correlation coefficient of variables $x_{i}$ and $x_{s}$;

$\mathrm{n}$ is used to designate the size of the sample;

$\sigma_{x_{i}^{2}}$ is the variance of variable $x_{1}$;

$\bar{x}_{i}$ is the average of the variable $x_{i}$;

$\sigma_{x_{i} x_{S}}$ is the covariance between $x_{i}$ and $x_{S}$;

$i, j, s$ are dummy indices used in summation terms.

\section{Absolute Error}

The absolute error of a function $W=F\left(x_{i}\right)$ is defined as

$$
\delta_{w}=\sum_{i=1}^{k}\left(\frac{\partial F}{\partial x_{i}}\right) \delta x_{i}
$$

where $\frac{\partial F}{\partial x_{i}}$ are evaluated from some given point $\left(x_{i}(0)\right)$. 
The relative error of a function $W=F\left(x_{1}\right)$ is defined as

$$
\frac{\delta w}{w}=\sum_{i=1}^{k}\left(\frac{\partial F}{\partial x_{1}}\right) \frac{\delta x_{i}}{w} .
$$

Probable Error

The probable error of a function, given an error $e_{x_{1}}$ in the measurement of each $x_{i}$, is defined as:

$$
E=\left[\sum_{i=1}^{k}\left(\frac{\partial F}{\partial x_{i}} e_{x_{i}}\right)^{2}\right]^{1 / 2}
$$

Mean of a Function

The mean of a function $W=F\left(x_{i}\right)$ is given as:

$\bar{W}=F\left(\bar{x}_{i}\right)+R$

Where $\mathrm{R}$ is equal to:

$$
R=\frac{1}{2} \sum_{i=1}^{k}\left(\frac{\partial^{2} F}{\partial x^{2}}\right) \frac{\sigma_{i}^{2}}{n}+\sum_{j=1}^{k-1} \sum_{s=j+1}^{k}\left(\frac{\partial^{2} F}{\partial x_{i} \partial x_{s}}\right) \frac{\sigma x_{1} x_{s}}{n} \cdot
$$

When $n$ becomes large $R$ tends to zero, leaving

$$
\overline{\mathrm{W}} \doteq \overline{\mathrm{F}}\left(\mathrm{x}_{\mathrm{i}}\right)
$$

as the general, approximating expression for the function mean where the variations are assumed small and normally distributed. No assumption of independence is made in $(A-5)$; if independence pertains then $(A-5)$ simplifies to 


$$
R=\frac{1}{2} \sum_{i=1}^{k}\left(\frac{\partial^{2} F}{\partial x_{i}^{2}}\right) \frac{\sigma_{x_{i}^{2}}^{2}}{n}
$$

\section{Variance of a Function}

The variance of a function $w=F\left(x_{1}\right)$ is defined by the equation

$$
\sigma_{F}^{2}=\sum_{i=1}^{k}\left(\frac{\partial F}{\partial x_{i}}\right)^{2} \sigma_{x_{1}}^{2}+2 \sum_{j=1}^{k-1} \sum_{s=j+1}^{k}\left(\frac{\partial F}{\partial x_{j}} \frac{\partial F}{\partial x_{s}}\right) r_{x_{j}} x_{s} \sigma_{x_{j}{ }_{s}}
$$

where $\sigma_{\mathrm{x}_{\boldsymbol{i}}}$ and $\sigma_{\mathrm{x}_{\mathrm{s}}}$ are standard deviations.

Covariance of Functions

Given that $U=F\left(x_{1}\right)$ and $V=G\left(x_{i}\right)$ are two functions their covariance is defined as:

$\sigma_{u v}=\sum_{i=1}^{k}\left(\frac{\partial U}{\partial x_{i}} \frac{\partial v}{\partial x_{i}}\right) \sigma_{x_{i}}^{2}+\sum_{j=1}^{k-1} \sum_{s=j+1}^{k}\left(\frac{\partial U}{\partial x_{j}} \frac{\partial v}{\partial x_{s}}+\frac{\partial U}{\partial x_{s}} \frac{\partial v}{\partial x_{j}}\right) r_{x_{j} x_{s}{ }^{\sigma} x_{j}{ }^{\sigma} x_{s}}$

\section{Systematic Error}

A systematic error is defined as a fixed deviation which is in each measurement of a variable in a particular sequence of measurements. Given $\mathrm{W}=\mathrm{F}\left(\mathrm{x}_{\mathbf{i}}\right)$, the systematic error is:

$$
|\delta W|=\sum_{i=1}^{k}\left|\begin{array}{ll}
\partial F & \delta x_{i}
\end{array}\right|
$$

The user of these formulas should keep in mind that they are approximations, and in strict mathematical terms the equality sign is not 1iterally true. Further, the underlying assumptions on which these formulas 
are based should be reviewed for compliance to statistical requirements in any problem of sensitivity analysis in which they are used.

The $x_{i}$ in the above equations are not restricted to variables; they can be thought of as parameters, coefficients, or input data for which a sensitivity response is to be measured. 
Part II: Statistics of the Index $w=x / y$.

The theory underlying the statistics of $w=x / y$ is presented in a thorough and exhaustive analysis by Fieller [6] which is far too difficult and lengthy to reproduce here. The general idea, based on [6], will be reviewed for those interested in the background of these approximations.

Following Fieller, the ratio $x / y$ is expanded by their variations, $\delta x$ and $\delta \mathrm{y}:$

Let $x=\bar{x}+\delta x$ and $y=\bar{y}+\delta y, \bar{x}$ is the expected value of $x ; \bar{y}$ is the expected value of $y$; expand as follows:

$$
w=\frac{\bar{x}+\delta x}{\bar{y}+\delta y}=\frac{\bar{x}}{\bar{y}}\left(1+\frac{\delta x}{\bar{x}}\right)\left[1-\frac{\delta y}{\bar{y}}+\frac{\delta y^{2}}{\bar{y}}-\frac{\delta y^{3}}{\bar{y}}+\ldots\right] \text {, }
$$

Then compute the $n$-th moment about zero of the distribution of $w$, from the equation for $\mathrm{Wn}^{\mathrm{n}}$. To obtain this value (based on a derivation by Merrill, see reference [6], page 436) retain the products of $(\delta x) r(\delta y) s$ as far as the eighth order, and take for their mean values the product-moments of the normal surface. This holds only for $|\delta y|<y$, but it is valid when applied to the interior of the probability contour. Merrill's values of the moments may be taken as the moments of the distribution of $w$ in a curtailed normal population. This provides, then, the basis for the formulas given in Section 3.3 .1 .1 , equaitons $(3.2)$ and $(3.3)$. 


\section{Appendix B}

Parametric Programming: Sensitivity Analysis

Parametric linear programming can involve the evaluation of six types of modifications. Given a typical linear program as:

$\begin{array}{ll}x>0 & y>0 \\ A x<d & A^{\prime} y>c \\ \max c^{\prime} x=z & \text { min } d^{\prime} y=z \\ \text { (Primal) } & \text { (Dual) }\end{array}$

In these formulations $A$ is the matrix of technical coefficients, $d$ is the vector of resources, $c$ is the vector of costs or profits, $x$ is the vector of allocations, and $z$ is the objective function, which is a scalar product. The symbol "'" indicates the transpose of the matrix $A$ or the vectors $c$ and $d$. Two properties which link the primal-dual solutions together are important to parametric sensitivity analysis. The first property states that the solution of the dual problem acts as an upper bound on the solution of the primal problem. The second property states that if $B$ is the basic matrix of the solution to the primal problem then the optimum solution of the dual is $Y=c B^{-1}$, where $c$ is the cost vector associated with the primal solution. The impact of a parametric sensitivity analysis on the integrity of these two properties should be assessed with regard to the consistency the modifications have with the primal-dual relationship. A parametric sensitivity analysis of a linear program may take any of the following modifications. 
The six possible modifications are:

(1) c changes by a discrete amount,

(2) d changes by a discrete amount,

(3) a single column of matrix A changes by some amount,

(4) a single row of A changes by some amount,

(5) a constant is added to the system, and

(6) a variable is added to the system.

These six cases are treated thoroughly by Simonnard 1 and the reader is referred to this text for an excellent exposition and analysis of these subjects.

Only the first modification, changes in the cost vector $c$, will be studied in this Appendix. The analysis entails calculating the bounds in which c may vary without altering the optimal solution. If the values of $c$, say $c_{i}$, are varied by a constant value $t$ then the objective function in the primal becomes $\left(c^{\prime}+t\right) x$. The parameter $t$ will appear in the tableau in the

${ }^{1}$ Simonnard, M., Linear Programming, Prentice-Hall, Inc., 1966, translated by William S. Jewe11, Chapter 7. 
last row and the simplex method will yield several inequalities on $t$. These inequalities define the interval on $t$ for which the optimal solution will not be altered. The objective function is then defined as $\left(c^{\prime}+t_{L}\right) x^{*}<z<\left(c^{\prime}+t_{u}\right) x^{*}$, where $x^{*}$ is the optimal solution. If $t$ is outside the interval $\left[t_{L}, \tau_{u}\right]$ then the optimal solution is changed.

If the problem is to study variations in the requirements vector $d$, the linear program may be recast in the dual form and variations in $d$ may be examined in the same manner as was done for $c$ in the explanation above.

Krekol develops a general theory for variations in $c$ or $d$. He defines $t_{L}$ and $t_{u}$ as characteristic points of the parameter $t$ and elaborates the conditions for which optimal solutions change as a function $t$. His analysis describes the solutions associated with the characteristic points of the $t$ domain, and develops a procedure for establishing upper and lower bounds on $t$ and the associated optimal solutions associated with a monotonic sequence of characteristic points.

${ }^{1}$ Kreko, B., Linear Programming, American Elsevier Co., Inc., 1968, Sections 4.6 and 12.3 


\section{Appendix C}

Catastrophe Flags and System Dynamics

The text of Section 6.4, Catastrophe Theory, is augmented here with greater detail on the dynamics of system stability as a function of changes in control parameters, and with a discussion of the catastrophe flags, which have a bearing on model dynamics, design and validation.

Figure $\mathrm{C}-1$ depicts the geometry of the cusp catastrophe associated with a parabolic force potential manifold. The response surface, $M$, is the folded sheet in three dimensions. Its projection, $C$, is the control parameter space, which is shown in Figure $\mathrm{C}-1$ with two parameters $(\mathrm{a}, \mathrm{b})$ and a cusp emanating from the point $P$. Four regions in the control space are marked as $R_{1}, R_{2}, R_{3}$, and $R_{4}$. Similarly in the manifold space are regions designated as $0_{0}, 0_{1}$, and $Q_{2}$ - The points $u$ and $v$ in $M$ are "jump" points at which the state variable jumps to only stable minimum values. Regions $Q_{1}$ and $O_{2}$ have minimum potential values. The region of the fold has maximum potential value and is unstable.

The discussion which follows on the catastrophe flags includes the general phenomena of system dynamics presented in Section 6.4 .

\section{Modality Flag}

Modality refers to the condition that the system has, in the case depicted in Figure C-1, two or more distinct physical states when the control parameters have values lying within the cusp-shaped regions. The projection of any point lying within the cusp-line boundary will pierce the manifold in three places. Al1 such points are, therefore, states of instability for the system. 


\section{Sudden Jump Flag}

A sudden change in the state of the system occurs when the state variable jumps from one sheet of the manifold to the other. In Figure C-1, this occurs when the control parameters describe a path from regions $R_{1}$ or $R_{2}$ to $R_{3}$ or $R_{4}$ where the path enters the cusp region and exits by crossing a cusp line. A sudden jump can occur when the control parameters move the state variable from $Q_{2}$ and $Q_{1}$ by traversing through the point $v$ or the cusp-line $\left(0_{0}, v\right)$. Moving the state variable from region $Q_{1}$ to $Q_{2}$ by crossing the cusp-line $\left(Q_{0}, u\right)$ causes a similar sudden jump to equilibrium in the $Q_{2}$ region. A catastrophic jump occurs when a smooth and continuous variation of control parameters causes a discontinuous change of state.

\section{Divergence Flag}

A divergence is said to occur when a smooth and continuous change in control parameters leads to a smooth and continuous change in the system state variable. This can be seen in Figure C-1. If the system state variable is in the region near $Q_{0}$ it can be moved into region $Q_{2}$ when the parameters follow a smooth path from $R_{1}$ to $R_{2}$. A similar movement can lead the state variable from $Q_{0}$ to $Q_{1}$ as the control parameters move along a path from $R_{1}$ to $R_{3}$ (or $R_{4}$ ) which does not traverse the cusp region. So by smooth transitions the state variable can be brought to divergent values, one in the region of $\mathrm{Q}_{2}$, the other in the region $\mathrm{Q}_{1}$.

\section{Hysteresis Flag}

This condition is included because it may have some bearing on the evaluation and behavior of modeling design. Hysteresis describes the situation in which if the path of the control parameters is reversed the path 
of the state variable is not necessarily reversed. Reversibility is possible if the parameter path does not exit from the cusp region. The system may be rendered unstable but jumps will not occur unless the parameter path exits from the cusp boundary.

Maxwe11 and Delay Rules

An interesting application of catastrophe theory is presented in Figure C-2. Two rules of behavior, or choices, are indicated, which assume that behavior may in some fashion perform as a system which seeks a condition of stable or minimum potential. If a policy is at point $\mathrm{P}_{\mathrm{O}}$ on an issue which is polarized around policies $\mathrm{P}_{1}$ and $\mathrm{P}_{2}$, the Delay rule invokes a smooth jump to policy $\mathrm{P}_{1}$ and the Maxwell rule invokes a discontinuous jump transition to policy $\mathrm{P}_{2}$. Under the Delay rule the jump is attended by a smooth, nondifferentiable change in the state variable which changes the policy in the direction which locally maximizes support. Under the Maxwell rule the jump is accompanied by a discontinuous change in the state variable which changes the policy to where the support is globally maximized.

The application of this idea lies in identifying regions of instability in system state variables, and being able to anticipate changes in the state variables by designing appropriate system response functions to signal system instability, or to detect that a change in policy is required to maximize effectness. 


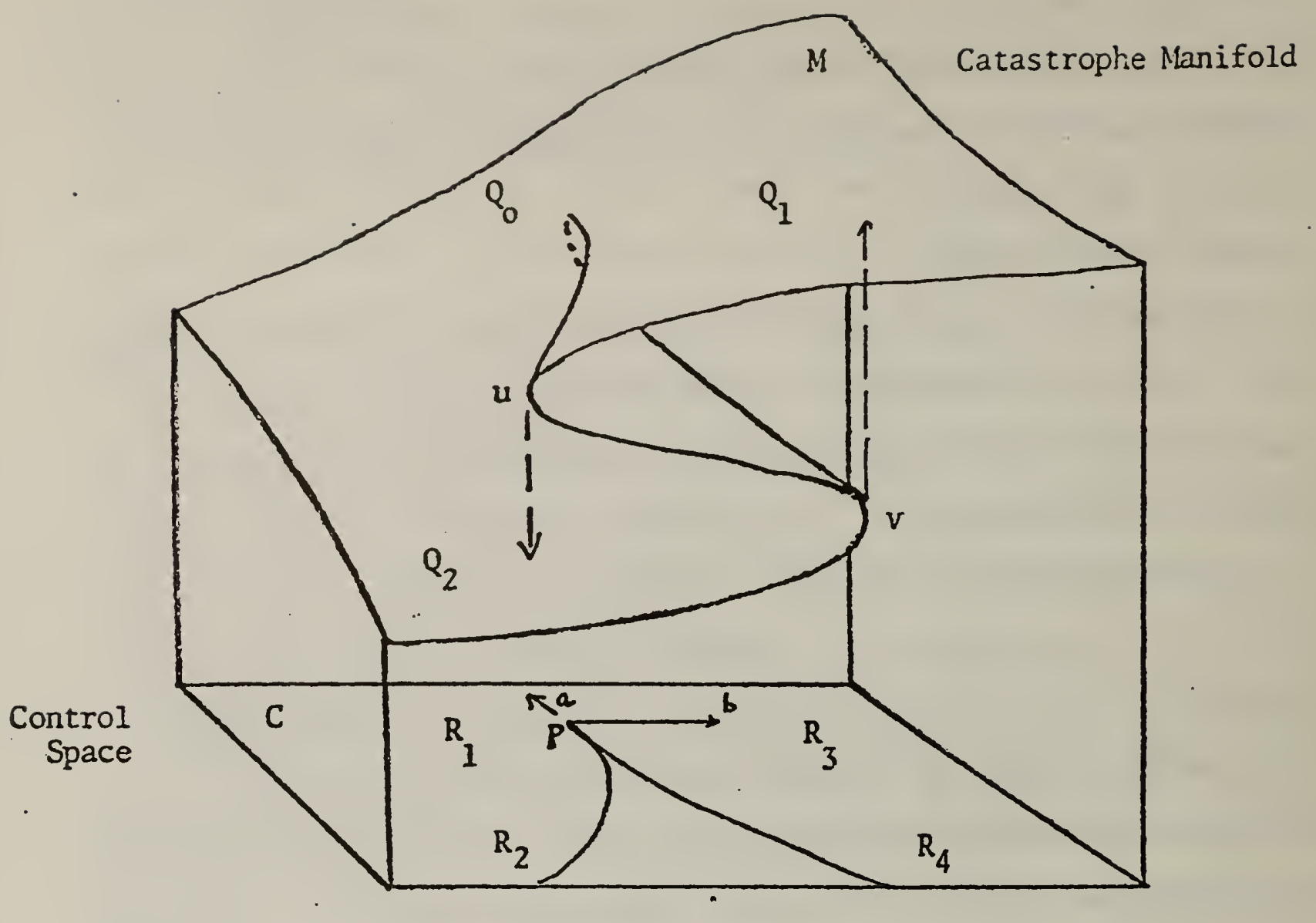

Figure C-1 : Catastrophe Cusp Geometry

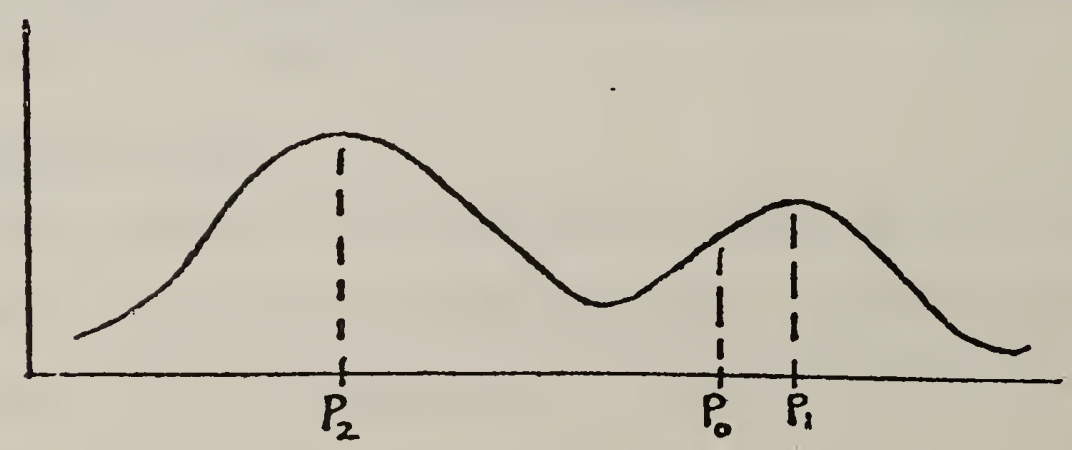

Figure C-2: Manwell and Delay Choices 


\section{REFERENCES}

1. Kelly, L. G., Handbook of Numerical Methods and Applications, Addison-Wesley, Introduction; 1967.

2. Stoer, J. and K. Bulirsch, Introduction to Numerical Analysis, Springer-Verlag, New York Inc., 1980 (English Version).

3. Campion, P. J., J. E. Burns, and A. Williams, A Code of Practice for the Detailed Statement of Accuracy, National Physical Laboratory, Department of Trade and Industry, 1973, Sections 2 and 3, and Appendices 1,3 and 4.

4. Kline, S. J. and F. A. McClintock, Describing Uncertainties in Single Sample Experiments, Mechanical Engineering, January 1953.

5. Dietrich, C. F., Uncertainty, Calibration and Probability, Wiley, 1973.

6. Fieller, E. C., The Distribution of the Index in a Normal Bivariate Population, Biometrica, Vol. 24, 1932.

7. Craig, C., On the Frequency Function of $x y$, The Annals of Mathematical Statistics, Volume VII, Number 1, March, 1936.

8. Ha11, I. J. and C. B. Sampson, Tolerance Limits for the Distribution of the Product and Quotient of Normal Variates, Biometrics 29, 109-119, March 1973.

9. Huntington, E. G., Frequency Distribution of Product and Quotient, The Annals of Mathematical Statistics, Volume X, 1939.

10. Curtiss, J. H., On the Distribution of the Quotient of Two Chance Variables, The Annals of Mathematical Statistics, Volume XII, pages 409-421 .

11. Buckingham, R. A., Numerical Methods, London, Pittman \& Sons, 1962. 
12. Ku, H. H., Notes on the Use of Propagation of Error Formulas, Journal of Research of the National Bureau of Standards-C. Engineering and Instrumentation, Vol 70C, No.4, October-December, 1966.

13. Deming, W. E., Statistical Adjustment of Data, John Wiley \& Sons, First Edition, 1948, Chapter III.

14. Weld, L. D., Theory of Errors and Least Squares, Macmillan, 1916, Chapter 8.

15. Wright, T. W., and J. F. Hayford, The Adjustment of Observations, Second Edition, Van Nostrand, 1906.

16. Pugh, E. M. and G. H. Winslow, The Analysis of Physical Measurements, Addison-Wesley, 1966.

17. Johnson, N. L. and H. Tetley, Statistics, Volume II, Cambridge University Press, 1950.

18. Nielsen, K. L., Methods in Numerical Analysis, Macmillan, 1964 , Chapter 1.

19. Goodwin, H. M., Elements of the Precision of Measurements and Graphical Methods, McGraw-Hill, 1920.

20. Hald, A., Statistical Theory with Engineering Applications, Wiley \& Sons, 1952.

21. Hildebrand, F. B., Methods of Applied Mathematics, Prentice-Hall, Inc., 1952, Section 2.4, Chapter 2.

22. McKay, M. D., W. J. Conover, and D. E. Whitman, Report on the Application of Statistical Techniques to the Analysis of Computer Codes, Los Alamos Scientific Laboratory, LA-NUREG-6526-MS, Informal Report, NRC-4. 
23. McKay, M. D. and R. J. Beckman, W. J. Conover, A Comparison of Three Methods for Selecting Values of Inputs in the Analysis of Output from a Computer Code, Technometrics, Vol. 21, Number 2, May, 1979.

24. Tocher, K. D., The Art of Simulation, Princeton, D. Van Nostrand, 1963.

25. Ford, A. G. H. Moore, and M. D. McKay, Sensitivity Analysis of Large Computer Models: A Case Study of the COAL2 National Energy Model, LA-7772-MS, Informal Report.

26. Tsukibayashi, S., Estimation of Variance and Standard Deviation Based on Range, Kep. Stst. App1. Res., JUSE, Vo1. 5, No. 2, 1958.

27. Bayes, A. J., Minimum Variance Sampling Technique for Simulation Models, Journal of Association for Computing Machinery, Volume 19, 1972, pp. 734741 .

28. Yamane, T., Statistics: An Introductory Analysis, Harper \& Row, Third Edition, 1973.

29. Stee1, R. G. D., and Torrie, J. H., Principles and Procedures of Statistics, McGraw-Hill, 1960.

30. Kenda11, M. and Stuart, A., The Advanced Theory of Statistics, Volume 2: Interference and Relationship, Chapter 27; Macmillan, Fourth Edition, 1979.

31. Scheppe, F. C. and J. Gruhl, Systematic Sensitivity Analysis Using Describing Functions, M.I.T. Energy Lab, Cambridge, Mass.

32. Alsmiller, J.r, R. G., et al., Adjoint Sensitivity Theory and its Application to LEAP Model 22C, Oak Ridge National Laboratory, ORNL/TM-7789.

33. Oblow, E. M., Sensitivity Theory from a Differential Viewpoint, Oak Ridge National Laboratory, Nuclear Science Engineering, Vo1. 59, page 187, 1976; also Nuclear Science Engineering, Vol. 65, page 428, 1978. 
34. Oblow, E. M., Sensitivity Theory for General Nonlinear Algebraic

Equations with Constraints, Nuclear Science Engineering, Vol. 65, No. 1, January, 1978.

35. Cacuci, D. G., C. F. Weber, E. M. Oblow, and J. H. Marable, Generalized Sensitivity Theory for Systems of Coupled Nonlinear Equations, Oak Ridge National Laboratory, NUREG/CR-1003, ORNL/NUREG/TM-349.

36. Cacuci, D. G., C. F. Weber, E. M. Oblow, and J. H. Marable, Sensitivity Theory for General Systems of Nonlinear Equations, Nuclear Science and Engineering, Vol. 75, 88-110, 1980.

37. Bartine, D. E., E. M. Oblow, and F. R. Mynatt, Radiation-Transport Cross-Section Sensitivity Analysis-A General Approach Illustrated for a Thermonuclear Source in Air, Nuclear Science and Engineering, Vo1. 55, $174-167,1974$.

38. Alsmiller, Jr., R. G. et al, Interim Report on Model Evaluation Methodology and the Evaluation of LEAP, Oak Ridge National Laboratory, ORNL/TM-7245.

39. Taha, H. A., Operations Research, An Introduction, MacMillan, 1971.

40. Wendell, R. E., A New Perspective on Sensitivity Analysis in Linear Programming: A Tolerance Approach, WP-448 KE, Graduate School of Business, University of Pittsburgh, Pittsburgh, PA, 1982.

41. Karplus, W. J., Error Analysis of Hybrid Computer Systems, Simulation, Vol. 6, No. 2, February, 1966.

42. Tomovic, R. and M. Vukobratovic, General Sensitivity Theory, American Elsevier, 1972. 
43. Burns, J. R., Error Analysis of Nonlinear Simulations: Applications to World Dynamics, IEEE Transactions on Systems, Man, and Cybernetics, Volume 5, 1975, pp. 331-240.

44. Rahman, N. A., A Course in Theoretical Statistics, Griffin London, 1968.

45. Cukier, R. I., H. B. Levine, and K. E. Shuler, Nonlinear Sensitivity Analysis of Multiparameter Model Systems, Journal of Computational Physics, Volume 26, 1-42, 1978.

46. Duffin, R. J., E. L. Peterson, and C. Zener, Geometric Programming, Wiley, 1967.

47. Wilde, D. J. and C. S. Beightler, Foundations of Optimization, Prentice-Hall, 1967.

48. Poston, T. an I. Stewart, Catastrophe Theory and its Application, Pitman, 1978.

49. Gilmore, R., Catastrophe Theory for Scientists and Engineers, John Wiley $\&$ Sons, 1981.

50. Zeeman, E. C., Catastrophe Theory: Selected Papers, 1972-1977, Addison-Wesley, 1977.

51. Sinha, D. K., Catastrophe Theory and its Applications, John Wiley \& Sons, 1981.

52. Poston, T., and A. E. R. Woodcock, Zeeman's Catastrophe Machine, Proc. Camb. Phil. Soc., 1973, Volume 74, page 217.

53. Alsmiller, Jr., R.G., J. Barhen, J. E. Horwedel, J. L. Lucius, J. D. Drischler, The Application of Adjoint Sensitivity Theory To A Liquid Fuels Supply Mode1, ORNL/TM-8850, September, 1983.

54. Silberberg, E., The Structure of Economics: A Mathematical Analysis, McGraw-Hill Co., 1978. 
NBS.114A (REV. 2.8C)

U.S. OEPT. OF COMM.

BIBLIOGRAPHIC DATA

SHEET (See instructions)

4. TITLE AND SUBTITLE

A Survey of Sensitivity Analysis Methodology

5. AUTHOR(S)

Robert G. Hendrickson

6. PERFORMING ORGANIZATION (If joint or other than NBS, see instructions)

7. ContracU Grant No.

NATIONAL BUREAU OF STANDARDS

DEPARTMENT OF COMMERCE

2. Performing Organ. Report Noy 3. Publication Date

\section{PUBLICATION OR} (1)

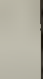

\section{(1)}

WASHINGTON, D.C. 20234

9. SPONSORING ORGANIZATION NAME AND COMPLETE ADDRESS (Street, City. State, ZIP)

10. SUPPLEMENTARY NOTES

Document describes a computer program; SF-185, FIPS Software Summary, is attached.

11. ABSTRACT (A 200-word or less factual summary of most significant information. If tocument includes a significant bibliography or literoture survey, mention it here)

This survey on the methodology of sensitivity analysis presents a general statement of the several broad categories of this discipline for the purpose of pulling together the various approaches and theory, to show the extent and sophistication of new techniques, special applications and the relation of sensitivity analysis to model evaluation.

12. KEY WORDS (Six to twelve entries; alphabetical order; capitalize only proper names; and separate key words by semicolons) error analysis; error propagation; model evaluation; model validation; sensitivity analysis

13. AVAILABILITY

XUnlimited

For Official Distribution. Do Not Release to NTIS

Order From Superintendent of Documents, U.S. Government Printing Office, Washington, D.C. 20402.

14. NO. OF

PRINTED PAGES

Order From National Technical Information Service (NTIS), Springfield, VA. 2216I

15. Price 

